\title{
DIFFUSION OF HEAVY IONS
}

IN THE

SOLAR CORONA

Yves Jean Alloucherie

April 1967

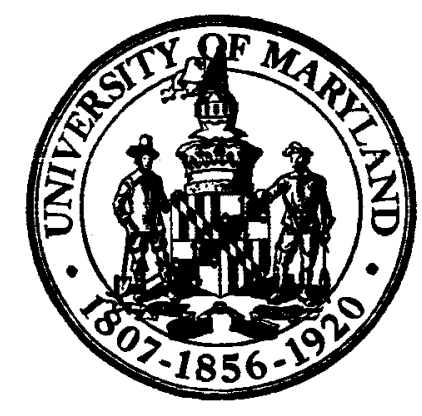

UNIVERSITY OF MARYLAND

DEPARTMENT OF PHYSICS AND ASTRONOMY COLLEGE PARK, MARYLAND

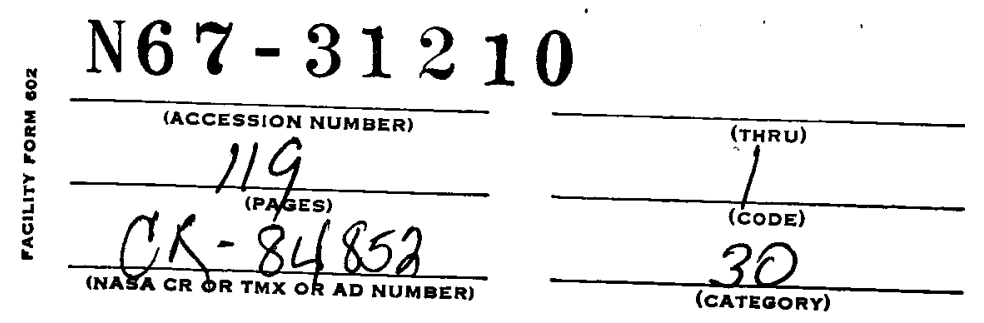




\section{APPROVAL SHEET}

Title of Thesis: Diffusion of Heavy Ions in the Solar Corona

Name of Candidate: Yves Alloucherie

Doctor of Philosophy, 1967

Thesis and Abstract Approved:

Derek A. 'Tidman

Research Professor

Institute for Fluid Dynamics and

Applied Mathematics

Date Approved: 
VITA

Name: Yves Jean Alloucherie.

Permanent address: 2707 Adams Mill Road, N. W. , Washington, D. C.

Degree and date to be conferred: Ph. D. , 1967.

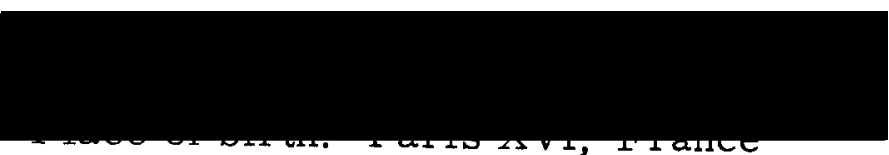

Secondary education: Baccalauréat, Séminaire de Québec, 1954.

Collegiate institutions attended Dates Degree Date of Degree

Université Laval 1954-55

$\begin{array}{llll}\text { University of Toronto } & 1955-59 & \text { B. A.Sc. } & 1959\end{array}$

$\begin{array}{llll}\text { University of Toronto } & 1959-61 & \text { M. A. } & 1960\end{array}$

$\begin{array}{llll}\text { University of Maryland } & 1961-67 & \text { Ph. D. } & 1967\end{array}$

Major: Plasma Physics.

Minors: Aerospace Engineering, Modern Physics.

Publication:

Non-Linear Constant - Profile Plane Waves in a Cold Plasma under an Applied Magnetic Field, J. Plasma Phys. 1, 55 (1967).

Positions held:

1959-1961 Demonstrator, Department of Physics, University of . Toronto, Toronto, Canada. 
1961-1963 Graduate Teaching Assistant, Department of Physics and Astronomy, University of Maryland, College Park, Maryland.

1963-1965 Scientist, Laboratory for Theoretical Studies, NASA Goddard Space Flight Center, Greenbelt, Maryland.

1965-1967 Scientist, Booz -Allen Applied Research, Inc., Bethesda, Maryland. 


\section{ABSTRACT}

Title of Thesis: Diffusion of Heavy Ions in the Solar Corona Yves Alloucherie, Doctor of Philosophy, 1967 Thesis directed by: Research Professor Derek A. Tidman

The steady-state diffusive motion of ionized atoms in the solar corona has been investigated theoretically, with special emphasis on relating the flow velocity and density of these ions to the flow properties of the ionized hydrogen background, under the assumption that the sun and its environment exhibits total spherical symmetry.

The basic approach taken in this study was to regard the ions as "test particles" immersed in, and interacting with, the electrons and protons making up the bulk of the corona. Two forms of the momentum equation were first derived for the ions, taking into account the effect of the following forces: the solar gravitational force, the kinetic friction force with the background electrons and protons, the electric force due to the gravity induced charge polarization of the electrons and protons, and the pressure gradient force. The first form of the equation is applicable to "cool" ions which have not yet reached thermal equilibrium with the "hot" background, while the second form assumes temperature equality between the ions and the 
protons. The momentum equation contains, as variable parameters, the density, velocity, and temperature of the electrons and protons. Since the ions have been assumed to be "test particles", they do not appreciably disturb the state of the ionized hydrogen background, and the above parameters may be obtained from existing dynamical models of the solar corona, which were obtained by neglecting the presence of the ions. Two such models have been specifically considered in this thesis: the one-fluid model of Parker (which postulates identical temperatures for the electrons and protons), and the two-fluid model of Sturrock and Hartle (which postulates different temperatures for the electrons and protons). The momentum equation was then combined with the equation of conservation of mass for the ions to give a first-order, non-linear differential equation for the flow velocity of the ions.

As a first step in the solution, an approximate, asymptotic relation was then derived, giving the physical conditions under which ionized atoms may either diffuse upward in the corona, remain at rest in equilibrium, or fall down toward the solar interior. A representative sample of ions satisfying the condition of upward convection was selected, and the velocity equation was solved numerically on a CDC 160-A computer for each one of these ions, using the model of Parker and the model of Sturrock and Hartle. These results were combined 
with the equation of conservation of mass to give, as a function of distance from the center of the sun, the relative abundance of these ions in the corona. It was found that the ions considered in the sample diffuse away from the sun with velocities somewhat lower than the velocity of the hydrogen background; the relative abundance of the ions, after a slight decrease near the bottom of the corona, increases gradually and slowly with height. 


\title{
DIFFUSION OF HEA VY IONS
}

\author{
IN THE
}

SOLAR CORONA

by

Yves Jean Alloucherie

Dissertation submitted to the Faculty of the Graduate School of the University of Maryland in partial fulfillment of the requirements for the degree of Doctor of Philosophy 


\section{ACKNOWLEDGEMENTS}

The author is deeply indebted to Dr. Derek A. Tidman, his thesis research advisor, for initially suggesting this problem, and for his continuous aid, guidance and encouragement given freely through the work which led to its solution. Special thanks are due to Dr. Wilmot N. Hess, Chief, Laboratory for Theoretical Studies, Goddard Space Flight Center, for extending the hospitality of his laboratory where this study was started. A number of other NASA scientists should also be thanked for helpful discussions, including Dr. M. P. Nakada and Dr. K. W. Ogilvie, also of the Goddard Space Flight Center.

The numerical solution of the diffusion equations was obtained on the CDC 160-A computer located in the Bethesda Office of Booz A Allen Applied Research, Inc. The author wishes to thank Mr. John F. Bernheisel, Manager of Computer Services, for making the computer available and for his general cooperation and assistance, Mr. Bryan Williams, who obtained the basic computer program, and Mrs. Rozann W. Saaty, for additional assistance.

This work was supported in part by the National Aeronautics and Space Administration under Grant NsG 220-62. This assistance is gratefully acknowledged. 


\section{TABLE OF CONTENTS}

Chapter

Page

ACKNOWLEDGEMENTS

LIST OF TABLES

$\mathrm{v}$

LIST OF FIGURES

LIST OF SYMBOLS

viii

I. INTRODUCTION. ................ 1

A. Definition of the Problem........... 1

B. Approach to the Problem.......... 8

C. Survey of Previous Work........... 14

II. DERIVATION OF THE EQUATION OF MOTION FOR THE HEAVY IONS. .........

III. THEORETICAL MODELS OF THE SOLAR CORONA..................... 50

A. Model of Parker.............. 52

B. Model of Sturrock and Hartle........ 58

IV. SOLUTION OF THE EQUATION OF MOTION FOR THE HEAVY IONS. . . . . . . . . . . . 64

A. General Discussion............ 64

B. Selection of Heavy Ions of Astrophysical Interest.............. 69

C. Model of Parker.............. 72

D. Model of Sturrock and Hartle........ 83

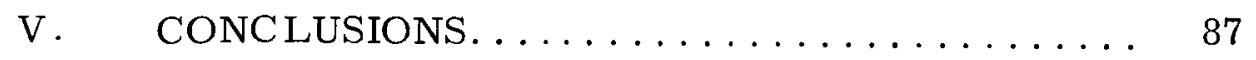


APPENDIX I. ..................... 89

APPENDIX II. ...................... 91

REFERENCES...................... 94 


\section{LIST OF TABLES}

Table

Page

I. Typical values of the collision frequency $r_{E}$ and the expansion rate $\mathbf{r}_{\mathbf{e}}$ in the corona

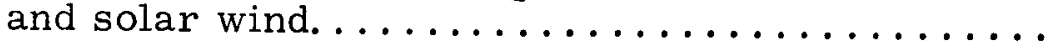

II. Numerical values of the coefficients $\boldsymbol{F}_{1}, \boldsymbol{F}_{2}, \boldsymbol{F}_{\mathbf{3}}$, $\boldsymbol{F}_{4}$, and $\boldsymbol{F}_{5}$ used to solve the diffusion 


\section{LIST OF FIGURES}

$\begin{array}{lll}\text { Figure } & \text { Page }\end{array}$

I. Physical Regions of the Sun and its

Environment....................... 2

II. Results of Jokipii for the Diffusion of

Alpha Particles in the Chromosphere........... 18

III. Theoretical Model of a Fully Ionized, Spherically Symmetric, Static Stellar

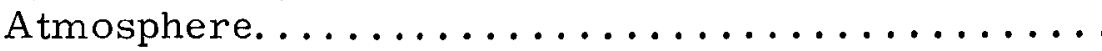

IV. The Family of Solutions of the Bernouilli

Equation (28), $\mathbb{U}$ versus $\pi$ (or $\boldsymbol{\pi}$ ), for an

Isothermal Corona and Various Values of

the Constant $L$ Designated on each Curve..........

V. Expansion Velocity $\boldsymbol{c}_{\bullet}(\mathrm{km} / \mathrm{sec})$ of an Iso-

thermal Corona as a Function of the Radial

Distance $r$ (in units of $10^{6} \mathrm{~km}$ ) from the

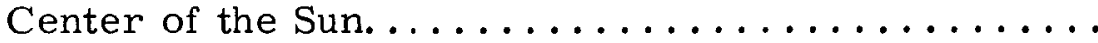

VI. The Flow Velocity $c_{\cdot}(\mathrm{cm} / \mathrm{sec}$, solid line)

and Electron Density $m\left(\mathrm{~cm}^{-3}\right.$, broken line)

as a Function of the Radial Distance (in units of $\pi_{0} \simeq 7 \times 10^{10^{\prime}} \mathrm{cm}$ ) from the Center

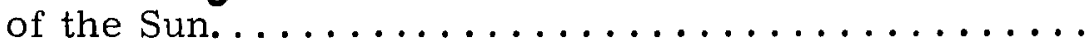

VII. The Electron Temperature $T_{1}\left({ }^{\bullet} \mathrm{K}\right.$, broken

line) and Proton Temperature $T_{2}\left({ }^{\circ} \mathrm{K}\right.$, solid

line) as a Function of the Radial Distance

(in units of $\boldsymbol{n}_{\boldsymbol{c}} \simeq 7 \times 10^{10} \mathrm{~cm}$ ) from the Center

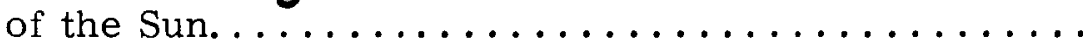

VIII. Computer Program Listing for the case of

${ }_{2} \mathrm{He}^{4}$ ions at $T_{3}=0$ and $T_{0}=1 \times 10^{6}{ }^{\circ} \mathrm{K}$, Ône-Fluid Model of Parker............... 
IX. Graph of the Flow Velocity of the Electrons, Protons and Ions as a Function of the Distance from the Sun. One-Fluid Model of Parker, $T_{1}=T_{2}=T_{0}=1 \times 10^{6} o_{K} \ldots \ldots \ldots \ldots \ldots$

X. Graph of the Flow Velocity of the Electrons, Protons and Ions as a Function of the Distance from the Sun. One-Fluid Model of Parker, $T_{1}=T_{2} \cdot T_{0}=2 \times 10^{6}{ }^{\circ} \mathrm{K} \ldots \ldots \ldots \ldots \ldots \ldots$

XI. Graph of the Relative Abundance of the Ions as a Function of the Distance from the Sun. One-Fluid Model of Parker, $T_{1}=T_{2}=T_{0}=1 \times 10^{6} o_{K} \ldots \ldots \ldots \ldots \ldots$

XII. Graph of the Relative Abundance of the Ions as a Function of the Distance from the Sun. One-Fluid Model of Parker, $T_{1}=T_{2}=T_{0}=2 \times 10^{6} o_{K} \ldots \ldots \ldots \ldots$

XIII. Graph of the Flow Velocity of the Electrons, Protons and Ions as a Function of the Distance from the Sun. Two-Fluid Model of Sturrock and Hartle, $T_{0}=2 \times 10^{6}{ }^{\circ} \mathrm{K} \ldots \ldots \ldots \ldots \ldots \ldots$

XIV. Graph of the Relative Abundance of the Ions as a Function of the Distance from the Sun. Two-Fluid Model of Sturrock and Hartle, $T_{0}=2 \times 10^{6} o_{K} \ldots \ldots \ldots \ldots \ldots \ldots$ 


\section{LIST OF SYMBOLS}

$$
\begin{aligned}
& a=M_{1} / 2 k T_{1} \\
& b=M_{2} / 2 k T_{2} \\
& \overrightarrow{\mathrm{B}} \quad=\text { magnetic field } \\
& \text { c } \quad=\text { speed of light } \\
& \vec{c}_{0} \quad=\left(c_{\theta_{\Omega}}, c_{\varphi,}, c_{\varphi \varphi}\right)=\left(\sum_{i=1}^{3} M_{i} M_{i} \vec{\mu}_{i}\right) / \rho=\text { flow velocity of } \\
& \text { solar corona } \\
& \text { e }=\text { electrical charge on an electron } \\
& \boldsymbol{e}_{i} \quad=\text { electrical charge on an individual particle of type } i \\
& \vec{E} \quad=\text { electric field } \\
& f_{i}=\text { distribution function for species } i \\
& f\left(m_{3} / m_{2}\right)=\text { diffusion coefficient due to pressure gradient } \\
& \vec{F}_{i} \quad=\left(F_{i_{\Omega}}, F_{i_{0}}, F_{i}\right)=\text { external force applied on an individual } \\
& \text { particle of type } i \\
& g(\Omega)=\text { acceleration of gravity } \\
& g_{3} \quad=\sum_{j=1}^{2} \int\left|\vec{v}-\vec{v}_{j}\right| f_{j}\left(\vec{v}_{j}\right) d^{3} \vec{v}_{j}
\end{aligned}
$$


$g\left(m_{3} / m_{2}\right)=$ diffusion coefficient due to thermal gradient

G $=$ universal gravitation constant

$G(\vec{n})=G(w)=\left(\frac{M_{1}+M_{3}}{M_{1}}\right) \frac{1}{w} \phi(w \sqrt{a})+\left(\frac{M_{2}+M_{3}}{M_{2}}\right) \frac{1}{w} \phi(w \sqrt{b})$

$h_{3} \quad=\sum_{j=1}^{2}\left(\frac{M_{j}+M_{3}}{M_{j}}\right) \int \frac{f_{j}\left(\vec{v}_{j}\right) d^{3} \vec{v}_{j}}{\left|\vec{v}-\vec{v}_{j}\right|}$

$h\left(m_{3} / m_{2}\right)=$ diffusion coefficient due to concentration gradient

$H(\vec{v})=H(w)=\frac{1}{4 a w}\left[4 w \sqrt{\frac{a}{n}} e^{-a w^{2}}+2\left(1+2 a w^{2}\right) \phi(w \sqrt{a})\right]$

$$
+\frac{1}{4 b-w}\left[4 w \sqrt{\frac{b}{n}} e^{-b-w^{2}}+2\left(1+2 b-w^{2}\right) \phi(w \sqrt{b})\right]
$$

$H=G M_{\odot} \rho_{0} / r_{0} p_{0}=G M_{0} M_{4} / 2 \pi_{0} k T_{0}=$ dimensionless

gravitational potential

$H_{i}=$ constant used in the definition of the polytrope index $\alpha_{i}$

$i=$ index used as a subscript after an other symbol and designating the applicable type of charged or neutral particles; i may take the value 1 (electrons), 2 (protons), 3 (heavy ions), or 4 (hydrogen atoms)

$I_{1}, \ldots . I_{5}=$ integrals

$\vec{j} \quad=\sum_{i=1}^{3} m_{i} \vec{\mu}_{i} r^{2} \quad=$ total particle flux density away from the sun 


$$
\begin{aligned}
& \mathrm{J}=\text { constant } \\
& \text { k = Boltzmann's constant } \\
& K=\text { constant } \\
& K_{1}=r_{0} g\left(r_{0}\right)\left(\frac{M_{2}}{4 k T_{0}}\right)\left[1-\frac{1}{2}\left(\frac{e_{3}}{e^{3}}\right) \frac{M_{2}}{M_{3}}\right] \\
& K_{2}=\frac{1}{2}\left(\frac{e_{3}}{l}\right) \frac{M_{2}}{M_{3}} \\
& K_{3}=\frac{2 \pi_{0} M_{0} e^{4}}{\left(k T_{0}\right)^{2}} \sqrt{\frac{\pi}{2}}\left(\frac{e_{3}}{l}\right)^{2}\left(\frac{M_{2}}{M_{3}}\right)^{2}\left(1+\frac{M_{3}}{M_{2}}\right) \\
& K_{4}=\frac{3}{2}\left(\frac{k T_{0}}{l^{2}}\right)^{3 / 2} \frac{1}{\sqrt{2 \pi M_{0}}} \\
& K_{5}=\frac{1}{4}\left(\frac{M_{2}}{M_{3}}\right) \\
& K_{6}=u_{0}=(u)_{r=r_{0}} \\
& t=\text { unit vector in the positive radial direction } \\
& M_{\mathbf{i}} \quad=\text { mass of an individual particle of type } i \\
& M_{E} \quad=\text { mass of Earth } \\
& M_{\odot} \quad=\text { mass of Sun } \\
& m_{i}=m_{0 i} \int f_{i} d \vec{v} \quad=\text { number density of species } i
\end{aligned}
$$




$$
\begin{aligned}
& m_{0 i}=\text { average number density of species } i \\
& N \quad=\sum_{i=1}^{3} m_{i} \quad=\text { total number density } \\
& N_{e} \quad=\text { number denoting the excess of electrons over protons } \\
& \text { outside of a spherical shell of radius } \pi \text { around the Sun } \\
& p_{i}=\operatorname{partial}(\text { scalar) pressure of species } i \\
& \overrightarrow{\vec{p}}_{i} \quad=M_{i} m_{0 i} \int \vec{w}_{i} \vec{w}_{i} f_{i} d \vec{v}=\text { pressure tensor of species } i \\
& \vec{r}=(r, \theta, \varphi)=(x, y, z)=\text { position vector in space about } \\
& \text { the center of the sun } \\
& \Omega_{0}=\text { reference radius } \\
& \Omega_{1}=\text { reference radius } \\
& \vec{R}=(R, \lambda, \mu) \quad=\text { dummy coordinates used in integrations } \\
& R_{\varphi} \quad=\text { universal gas constant } \\
& R_{\odot} \quad=\text { radius of sun } \\
& t=\text { time } \\
& T_{i} \quad=\text { temperature of species } i \\
& T=\text { temperature }
\end{aligned}
$$


$\vec{\mu}_{i}=\left(\mu_{i_{1}}, \mu_{i_{\theta}}, \mu_{i}\right)=\left(m_{0 i} \int_{f_{i}} \vec{v} d \vec{v}\right) / m_{i}=$ flow velocity of species $i$ relative to the sun

$\vec{u}_{i} \quad=\left(u_{i_{\Omega}}, u_{i_{0}}, u_{i_{p}}\right)=\left(m_{0 i} \int f_{i} \vec{w} d \vec{v}\right) / m_{i} \quad$ = flow velocity of species $i$ relative to the moving solar corona

$\vec{u}_{i, j}=\vec{u}_{i}-\vec{u}_{j}$

$U_{1}^{2}=$ constant

$U_{2}^{2}=$ constant

$u=c_{0} / \sqrt{2 p_{0} / \rho_{0}}=$ dimensionless velocity of the solar corona

$\vec{v} \quad=\left(v_{n}, v_{\theta}, v_{\varphi}\right)=$ velocity of an individual particle relative to the sun

$\vec{v}_{\mathbb{D}} \quad=$ drift velocity

$\vec{V}=\left(V_{\lambda}, V_{\theta}, V_{\varphi}\right)=$ velocity variable used in integrations

$V \quad=\mu_{3} / \sqrt{2 p_{0} / \rho_{0}}=$ dimensionless flow velocity of the heavy ions

$\vec{w} \quad=\left(w_{1}, w_{0}, w_{p}\right)=\vec{v}-\vec{c}_{0} \quad=$ velocity of an individual particle relative to the moving solar corona 


$$
\begin{aligned}
& \vec{w}_{i} \quad=\left(w_{i_{\Omega}}, w_{i_{0}}, w_{i_{\varphi}}\right)=\vec{v}-\vec{\mu}_{i} \quad=\text { velocity of an individual } \\
& \text { particle relative to the moving species } i \\
& X, Y, Z=\text { numbers giving the fractional composition of the solar } \\
& \text { interior } \\
& \alpha_{i} \quad=\text { polytrope index for species } i \\
& \beta_{i}=\text { constant } \\
& r=\frac{N_{\rho} e}{r^{2}}\left(\frac{e}{M_{4}}\right) \\
& \Gamma_{3}=\frac{4 \pi e^{4}}{M_{3}^{2}}\left(\frac{l_{3}}{e}\right)^{2} \log \Lambda=\frac{4 \pi e^{4}}{M_{3}^{2}}\left(\frac{e_{3}}{e}\right)^{2} \log \left[\frac{3}{2}\left(\frac{k T_{1}}{l^{2}}\right)^{3 / 2} \frac{1}{\sqrt{2 \pi M}}\right] \\
& \delta_{i} \quad=\text { constant } \\
& \epsilon \quad=\quad \sum_{i=1}^{3} e_{i} m_{i}=\text { total electric charge density } \\
& 5=r / r_{0}=\text { dimensionless radius } \\
& \eta \quad=\left\{1-\left(\frac{l_{3}}{l}\right) \frac{M_{2}}{M_{3}}\left[\frac{1}{1+T_{2} / T_{1}}\right]\left[1+\frac{c_{l}}{g(\lambda)} \frac{d c_{0}}{d r}\right]\right\} \\
& \vec{\theta}=\text { unit vector in the direction of increasing } \theta \\
& X_{i}=\text { thermal conductivity of species } i \\
& \text { x } \quad=\text { integer used in summations }
\end{aligned}
$$




$$
\begin{aligned}
& \lambda_{\boldsymbol{D}} \quad=\text { radius of Debye Sphere } \\
& \Lambda \quad=\frac{3}{2}\left(\frac{k T_{1}}{l^{2}}\right)^{3 / 2} \frac{1}{\sqrt{2 \pi M}} \\
& \mu_{i}=M_{i} / M_{4}=\text { atomic weight of a particle of species } i \\
& r=n / m_{0}=\text { dimensionless total number density of the } \\
& \text { solar corona } \\
& r_{\mathbf{l}}=\text { expansion rate of the solar atmosphere } \\
& r_{\mathbf{E}}=\text { collision frequency for energy exchange between } \\
& \text { electrons and protons } \\
& \pi=\text { distance from the center of the Sun, in units of GMogo/ } p_{0} \\
& \rho \quad=\sum_{i=1}^{3} M_{i} M_{i}=\text { total mass density } \\
& \rho_{i}=m_{i} M_{i}=\text { mass density of species } i \\
& \sigma_{i}=M_{i} \boldsymbol{l}_{i}=\text { electric charge density of species } i \\
& \sigma \quad=\sum_{i=1}^{3} m_{i} l_{i}=\text { total electric charge density } \\
& \vec{\varphi} \quad=\text { unit vector in the direction of increasing } \varphi \\
& \Phi \quad=\text { gravitational potential }
\end{aligned}
$$




$$
\begin{array}{ll}
\phi(x) & =\frac{2}{\sqrt{\pi}} \int_{0}^{x} e^{-y^{2}} d y=\text { error integral } \\
\Psi \quad & =\text { electrostatic potential } \\
\Omega \quad & =\text { angular velocity of the sun about itself }
\end{array}
$$

The subscript after a variable designates the value taken by that variable at the reference radius $r_{0}$, except in the case of $c_{0}$ defined above. 


\section{INTRODUCTION}

A. Definition of the Problem

The problem discussed in this thesis is concerned with a theoretical treatment of the dynamical motion of heavy ions in the solar corona. The expression "heavy ions" denotes any charged particles of astrophysical interest more massive than electrons or protons. The problem has three specific objectives: to discover whether or not such heavy particles will move away from the sun or will remain permanently trapped in its gravitational field, to obtain the flow velocity of the ions as they diffuse through the solar corona, and to find out how their relative abundances vary from point to point in the space around the sun. In order to justify this work, a brief review of the solar physics pertaining to the problem, as well as a number of important general assumptions, will now be given.

Most textbooks on solar physics divide the sun and its environment into a number of distinct physical regions: the solar interior, the photosphere, the chromosphere, and the corona which, extended, becomes the solar wind (Kuiper, 1958). These are shown in Figure 1 on page 2. The often quoted "solar radius" $R_{\odot}$ actually refers to the radius of the outer edge of the photosphere, the "sharp" visible solar 


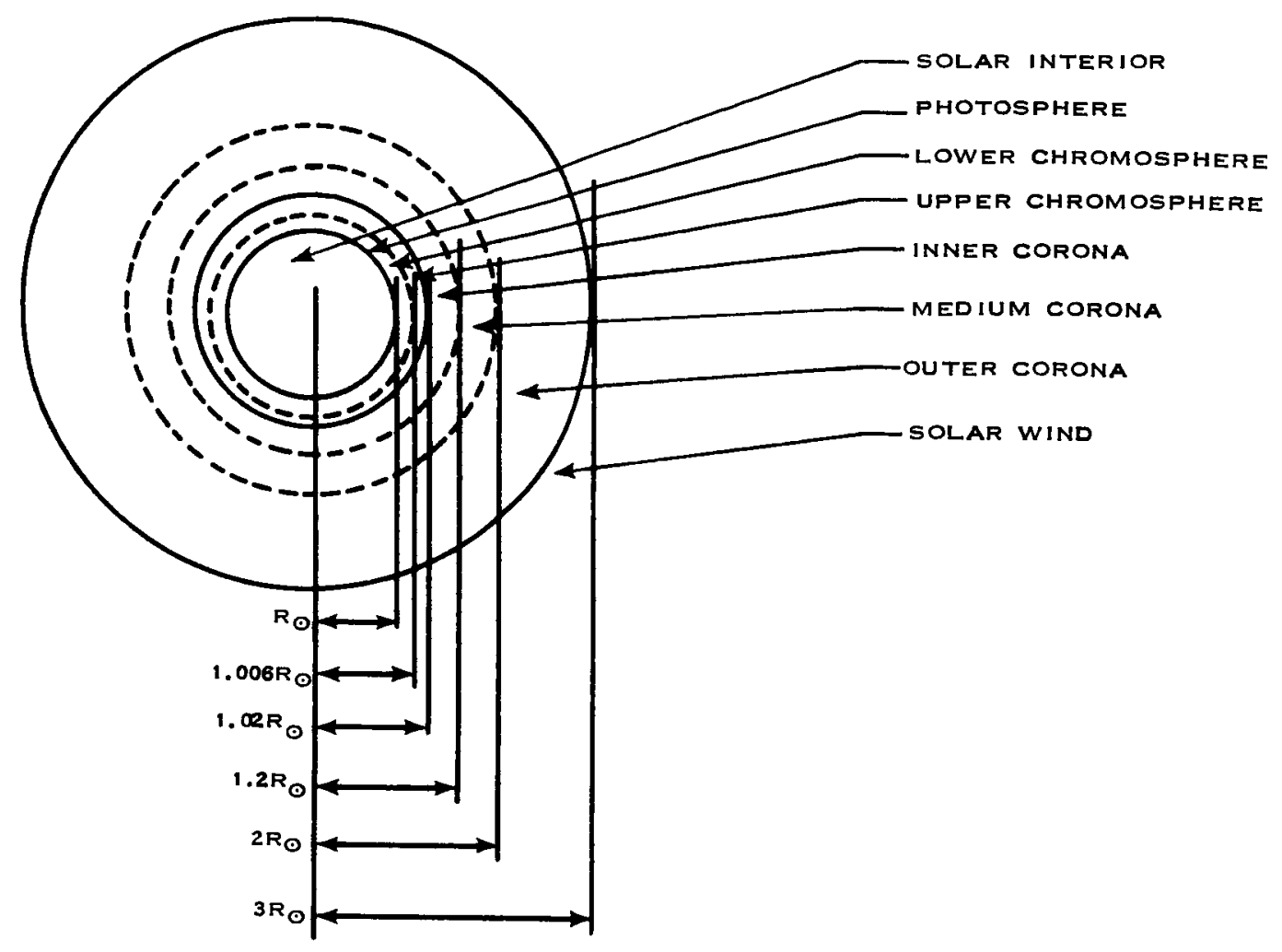

Figure I. Physical Regions of the Sun and its Environment

disk. In reality, the photosphere has a small thickness of its own, but this only amounts to about $0.001 R_{\odot}$. The chromosphere, divided between a lower and an upper region, reaches a height of about $2 \times 10^{9}$ $\mathrm{cm}$ above the solar radius $R_{0}$ and represents the main transition region in temperature between the comparatively cool photosphere $\left(T \sim 4500^{\circ} \mathrm{K}\right)$ and the much hotter lower or inner corona $\left(T \sim 1 \times 10^{6}{ }^{\circ} \mathrm{K}\right)$. 
The corona is itself divided into an inner corona, a medium corona, and an outer corona. According to Kuiper, the outer corona ends at $r=3 R_{0}$, where it then becomes synonymous with the solar wind, which extends to infinity.

The physical and chemical composition of the sun and its environment may be investigated theoretically or experimentally.

In view of the opacity of the photosphere, the composition of the solar interior has only been studied theoretically, since the only relevant quantities which may be measured experimentally are its mass, its volume, and its total energy output. Detailed physical and chemical information may still be obtained by applying the theory of stellar interiors to the sun. The fundamental equations describing the state of the solar interior have been solved under the assumption that one gram of substance making up the solar interior consists of a mixture of $X$ gram of hydrogen, $Y$ gram of helium, and $Z$ gram of all other, heavier elements (Schwarzschild, 1946; Harrison, 1948; Keller, 1948; Epstein, 1961; Kuiper, 1958); a number of different models were developed. Typical results were those obtained by Epstein: $X=0.82$, $Y=0.17, Z=0.01$. The solar interior therefore consists, by mass, primarily of hydrogen, with a small fraction of helium and traces of other elements. 
The composition of the solar atmosphere (photosphere, chromosphere, corona) has been extensively investigated experimentally, for instance by studying the Fraunhofer lines of the solar electromagnetic spectrum. According to Flammarion (1964), 21,835 such lines have been observed, varying in wavelength between $2975 \AA$ and $10218 \AA$; these have allowed astronomers to identify the characteristic spectra of most of the elements known on earth. Furthermore, the intensities of these spectral lines has provided information on the quantitative analysis of the solar atmosphere. The main result of the chemical analysis of the solar atmosphere is again the enormous preponderance of hydrogen. The abundance of helium is estimated to be something like 4 or 5 helium atoms for each 100 atoms of hydrogen.

In view of these results, it should now be clear that the dynamical properties of the solar corona may, as a first approximation, be investigated by assuming that it consists of ionized hydrogen only. This was done by a number of researchers who showed that the solar atmosphere is not static, but continuously expands away from the sun in all directions to become, far away from the sun, the solar wind. Two especially significant theoretical models of such a simplified solar corona and solar wind, derived by Parker (1963) and Sturrock and Hartle (1966), will be described in detail in Chapter III. 
The next logical step in obtaining a more complete physical description of the dynamics of all the charged particles making up the solar corona is to investigate the behavior of the heavier elements (helium and others) which have so far been ignored. In view of the inherent complexity of such a multi-fluid system, the scope of the problem treated in this thesis, previously defined by the three objectives stated at the beginning of this chapter, will be narrowed by making the following simplifying assumptions:

(1) We will limit our attention to the steady, time - independent, continuous motion of particles away from the sun. Purely transient phenomena, such as solar flares, coronal disturbances over sunspots, and prominences, which will perturb this steady motion, will not be considered.

(2) The complex phenomenon of solar rotation will also be neglected. The sun is not a rigid body and its angular velocity is greater at the equator than near the poles; in addition, there are indications that the angular velocity of the solar atmosphere varies with height above the sun's surface. However, this assumption may be justified by noting that, everywhere in the corona, the centripetal acceleration on a particle due to solar rotation is much smaller than the gravitational acceleration:

$$
\Omega^{2} r \ll g(r)
$$


where:

$\Omega$ (angular velocity of the sun) $\sim 3 \times 10^{-6}$ radians $/ \mathrm{sec}$ $r \sim 2 \times 10^{11} \mathrm{~cm}$ $g(r) \sim 3 \times 10^{3} \mathrm{~cm} / \mathrm{sec}^{2}$ at $\Omega \sim 2 r_{0}$ may be regarded as typical values.

The two previous assumptions will have the effect of giving complete spherical symmetry to our problem, which will therefore be reduced to a simpler one-dimensional case, with the radius $\pi$ as the only independent variable.

(3) The division of the sun and its environment into a number of layers, as shown previously in Figure I, is really too detailed for our purpose since we are primarily interested, in this problem, in the motion of the heavy ions in the corona and solar wind only; we are not so such interested in the complex convective processes taking place in the chromosphere. The following simpler model will therefore be adopted from now on, consisting of: a central spherical core, with an external reference radius $\boldsymbol{r}_{0}\left(\boldsymbol{r}_{\bullet}>\boldsymbol{R}_{\bullet}\right)$, combining together the solar interior, the photosphere, the chromosphere, and part of the lower corona; and a spherical shell external to this central core, combining together most of the corona and the solar wind. This agrees 
with the basic model adopted by Parker, who selected the value $\Omega_{0}$ $=10^{11} \mathrm{~cm}$.

(4) It will later be shown in Chapter III that the velocity predicted by Parker and Sturrock and Hartle for the electrons and protons making up the bulk of the solar wind keeps on increasing continuously with increasing distances from the sun: the solar wind has therefore no true "coasting velocity", although the rate of increase of that velocity decreases with increasing distances from the sun. It would therefore seem that, to obtain a mathematically complete solution, the diffusion velocity of the heavy ions would have to be obtained for $\pi_{0} \leqslant \pi<\infty$. In practice however, we are primarily interested in the region of the solar system between the sun and the earth. If the flow velocity of the solar wind electrons or protons is computed at the earth's orbit around the sun $\left(\pi \sim 150 \pi_{0}\right)$ and is used as a "reference velocity", it will be found that most of this velocity is reached by the solar wind when it is still much closer to the sun. For instance, typically, $50 \%$ to $60 \%$ of this reference velocity will be reached for $r=5 r_{0}$, which will be taken as the upper limit of integration in our calculations.

(5) Finally, numerical calculations will not be made for all the charged particles observed in the solar corona, but only for a selected sample of special interest. 


\section{B. Approach to the Problem}

In order to describe completely the flow properties of a typical kind of heavy ions (such as, for example, the Fe XV ions) in the solar corona, the motion of each such individual ion should be known. Let us suppose that we isolate and identify a typical Fe XV ion, and attempt to set up and solve the equation describing its motion in space. This will require a knowledge of all the forces acting on that "test particle": there will be a gravitational force due to the attraction of the central solar core (described previously in Section A of this chapter) and all the other particles in the solar atmosphere, an electric and a magnetic force due to the general background values of the se field quantities, and friction forces due to the collisional interactions between the ion and all the other particles in the corona. These other particles will include not only the electrons and protons which make up the bulk of the background material, but also all the other ions ( $F e X, F e X I$, Ni XVI, Ca XII, etc.) and, finally, all the Fe XV ions except the test particle. The exact description of the motion of a single test particle would therefore require, as a prerequisite, a knowledge of the instantaneous position and velocity of all the other particles in the solar corona. Since this information is not given explicitly, this approach 
would only lead to an unmanageable system of coupled integro-differential equations of motion. However, it may clearly be seen from intuition that such an exact dynamical description of our system is really unnecessary, since it has already been noted in Section $A$ above that sufficient spectroscopic evidence exists to show that the general coronal abundances of helium and heavier elements are very small compared to the abundance of hydrogen.

A simplified and more realistic approach to solve the problem will now be presented. This will be based on the following assumptions:

(1) Interactions between different species of heavy ions (such as Fe XV and Ca XII) will be neglected.

(2) Interactions between different ions of the same species will be neglected.

(3) The perturbing effects of the ions on the electrons and protons will be small enough so that the results obtained by other researchers (see Chapter III) for the flow of a coronal gas consisting of ionized hydrogen only will remain unchanged.

It is then possible, with these additional assumptions, to uncouple the various equations of motion at the start and to consider each ionic species independently of the others. 
The actual solution will be obtained in the following way:

(1) In Chapter II, two forms of the equation of motion describing the dynamical behavior of an arbitrary ion in the solar corona will be derived:

(a) The first form of this equation will be obtained by postulating that $T_{3}$, the temperature of the ions, is much lower than $T_{1}$ and $T_{2}$, the temperatures of the electrons and protons:

$$
\begin{aligned}
& T_{3} \ll T_{1} \\
& T_{3} \ll T_{2}
\end{aligned}
$$

(Note that we do not necessarily assume that $T_{1}=T_{2}$.) It will later be shown in Chapter IV that this postulate is valid in the lower regions of the corona, where the ions, rising from the much cooler chromosphere, did not yet have time to reach thermal equilibrium with the much hotter ionized hydrogen background.

(b) The second form of this equation will be obtained by postulating that the ions have reached thermal equilibrium with the coronal protons:

$$
T_{3}=T_{2}
$$

Here again, we do not necessarily assume that $T_{1}=T_{2}$. It will later be seen in Chapter III that Parker, in deriving his ionized hydrogen 
corona model, postulated that $T_{1}=T_{2}$ everywhere; on the other hand, Sturrock and Hartle, in the derivation of their model, relaxed this prescription and came to the conclusion that $T_{1}>T_{2}$ everywhere, except at $\boldsymbol{\Omega}=\boldsymbol{R}_{0}$ where $T_{1}=T_{2}$. It will also later be shown that both these models are based on the assumption that $m_{1} \simeq m_{2}$ everywhere. Finally, $M_{1} \ll M_{2}$ so that kinetic energies transformed into heat during an electron-ion collision will be, in general, much lower than the energies involved during a proton-ion collision. It therefore follows that the ions will eventually reach thermal equilibrium with the protons rather than with the electrons, which explains the prescription $T_{3}=T_{2}$ used in the derivation of the second form of the equation of motion. This form will later be shown to be valid in the outer regions of the corona.

Both forms of the equation of motion will depend parametrically on such quantities as the flow velocity of the electrons and protons, their number densities, their temperatures, as well as on basic constants such as the charge and mass of the ion, etc.

(2) In Chapter III, the theoretical models of an ionized-hydrogen corona derived by Parker and Sturrock and Hartle will be described and reviewed. Special emphasis will be placed on showing how these models 
may be used to compute the flow velocity, density, temperature and pressure of the electrons and protons as a function of position in space.

(3) Chapter IV, which deals with the solution of the equation of motion derived previously in Chapter III, is divided into four sections:

(a) A general discussion will first be given. This will include the derivation of an approximate asymptotic relationship giving the conditions under which a specific ion will either move away from the sun, will remain stationary in equilibrium in the corona, or will fall back deeper toward the solar interior.

(b) It has already been pointed out that the spectroscopic emission lines originating in the corona are so numerous that most elements found on the earth have been identified in the solar atmosphere. Many of these elements, and especially the heavier ones, may be ionized in many states so that the total number of charged particle species is very great. The purpose of this section is to choose, from this large collection, a representative and reasonable sample of ions to be actually used in calculations. 
(c) Four sets of solutions will then be obtained in the next two sections, corresponding to the two different models of the corona and to the two thermal possibilities for the ions (before and after thermal relaxation with the protons). The basic approach will first consist in combining together the equation of motion with the equation of conservation of mass to obtain a nonlinear differential equation in the velocity only. This equation will then be integrated numerically on a computer from $\pi=\pi_{0}$ to $\pi=5 \boldsymbol{r}_{0}$, using a relaxation method (described in detail later) to obtain the correct boundary value for the velocity at $\boldsymbol{r}=\Omega_{0}$. After the velocity has been obtained, the equation of conservation of mass will again be used to find how the abundances of the ions vary throughout the corona. 


\section{Survey of Previous Work}

A brief review of the theoretical results directly related to the diffusion of heavy ions in the solar corona obtained previously by other a uthors will now be presented. No attempt will be made to review experimental work at this point; this will be done later.

It has already been mentioned that the theoretical models of the corona derived by Parker (1963) and Sturrock and Hartle (1966) are based on the assumption that the solar atmosphere consists of ionized hydrogen only. In the fourth chapter of his book, where he showed that the solar corona cannot be static but must expand outward continuously, Parker however presented a brief discussion concerning the relative abundances of heavy ions as a function of height in a hypothetical static solar atmosphere; even though this atmosphere is, strictly speaking, not static, but expanding, Parker's remarks may be regarded to have some validity near the base of the corona, since it will later be shown (in Chapter III) that the theoretical radial expansion velocities in that region are quite small, so that "quasi-static" conditions may possibly be assumed to prevail there. He first showed, using elementary statistical physics, that, in a state of complete static 
equilibrium (and assuming that $T_{1}=T_{2}=T_{3}$, or thermal equilibrium between the ionized hydrogen and the ions), the abundances of heavy ions, relative to the ionized hydrogen background, will decrease rapidly with height, since they have a scale height of the order of $k T_{3} / M_{3} g(r)$, and $M_{1} \ll M_{2} \ll M_{3}$. Furthermore, he went on to say that if this idealized "static" model corona is stirred vigorously for a while to produce a momentary homogeneous abundance of heavy ions, the return to equilibrium will be fairly rapid and the ions will settle down quickly again: as an illustration, Parker mentioned the following case of the downward drift of an alpha particle in a hydrogen corona of density $\boldsymbol{M}$, temperature $\boldsymbol{T}$, and gravitational acceleration $\boldsymbol{g}$. The mean downward drift velocity $\boldsymbol{N}_{\mathbb{D}}$ for the alpha particle between collisions will be $1 / 2 \mathbf{g t}_{2} \mathrm{He}^{+} *$, where $t_{2} \mathrm{He}^{+}$is the collision time for the alpha particles. Near the base of the corona, $m \sim 10^{9}$ particles $/ \mathrm{cm}^{3}$ and $v_{p} \sim 1 \mathrm{~km} / \mathrm{sec}$ if $T \sim 2 \times 10^{6}{ }^{\circ} \mathrm{K}$; at an altitude of $3 \times 10^{5} \mathrm{~km}$ in the

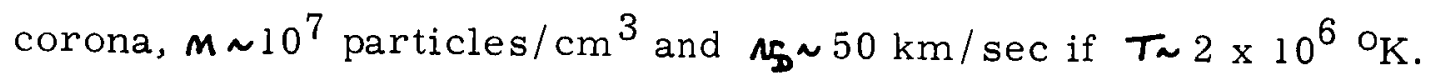
It therefore appears from these figures that vigorous stirring or expansion will be necessary to convect the ions upward and away from

* This neglects the effect of the upward electric field on the alpha particle due to the gravity induced charge separation effect between the electrons and protons. This will be discussed at length in Chapter II. 
the sun, and it becomes clear that a quantitative solution to the problem will depend on the balance between the gravitational, electric, magnetic and friction forces acting on each ion.

The next step was taken by Jokipii (1967), who published a brief account of his work in "The Solar Wind", edited by Robert J. Mackin, Jr. and Marcia Neugebauer. The basic conceptual approach taken by Jokipii was similar to the approach followed in this thesis. After making the basic assumption of steady-state motion and perfect spherican symmetry, he proceeded to study the dynamical motion of a tenuous gas of heavy charged particles injected into a background of fully ionized hydrogen flowing away from the sun. The rest of his treatment was however different. He assumed that the mean free paths in the corona were small enough for the diffusion equation of ordinary fluid mechanics to be valid. The radial component of this equation (as given by Jokipii but rewritten in our notation) was stated without proof in the following form:

$$
\begin{aligned}
\frac{\mu_{2}-\mu_{3}}{\mu_{2}}=\frac{T^{3 / 2}}{m_{2} \mu_{2} \Omega^{2}}\left\{f\left(M_{3} / m_{2}\right)\right. & -g\left(M_{3} / m_{2}\right) \frac{\Omega^{2}}{R_{0}^{2}} \frac{d T}{d r} \\
& \left.+h\left(m_{3} / m_{2}\right) \frac{r^{2}}{R_{0}^{2}}\left[\frac{T}{1-\left(\frac{\mu_{3}-\mu_{3}}{\mu_{2}}\right)}\right] \frac{d}{d r}\left(\frac{\mu_{2}-\mu_{3}}{\mu_{2}}\right)\right\}
\end{aligned}
$$

where $f\left(m_{3} / m_{2}\right), g\left(m_{3} / m_{2}\right)$, and $h\left(m_{3} / m_{2}\right)$ represent the diffusion coefficients due to pressure, thermal, and concentration gradients 
respectively and are slowly varying functions of $m_{3} / m_{2}$; although no specific mention is made to that effect in his paper, Jokipii apparently assumed complete thermal equilibrium between the electrons, protons, and heavy particles at all times:

$$
T_{1}-T_{2}=T_{3}=T
$$

The above diffusion equation was combined with the continuity equation and solved to obtain values of $\left(\mu_{2}-\mu_{3}\right) / \mu_{2}$ and $\boldsymbol{M}_{3} / \boldsymbol{M}_{2}$ as a function of the height $\left(\Omega-R_{\odot}\right)$ above the photosphere, using alpha particles as the heavy ions; two sets of values were obtained for $\boldsymbol{m}_{\mathbf{3}} / \boldsymbol{m}_{\mathbf{2}}$, corresponding to the cases of perfect mixing and no mixing between the alpha particles and the ionized hydrogen background. Jokipii does not specifically state what functional relationships he assumed to describe $T=T(\Omega)$ and $\mu_{2}=\mu_{2}(\Omega)$ in order to find $\mu_{3}=\mu_{3}(\Omega)$; all his results were presented graphically only and are reproduced in Figure II on page 18 of this thesis. A notew orthy aspect of the se results lies in the fact that the lower integration limit selected by Jokipii, $\boldsymbol{\Omega}=\boldsymbol{R}_{\boldsymbol{\odot}}$ (radius of the photosphere) is lower than the value $\boldsymbol{\Omega}=\boldsymbol{r}$. selected in this thesis: this explains the large variations in $T,\left(\mu_{2}-\mu_{3}\right) / \mu_{2}$ and $\boldsymbol{m}_{3} / \boldsymbol{m}_{\mathbf{2}}$ near the lower integration limit, through the chromospheric transition layers. On the other hand, his range of integration $(\sim 60,000$ $\mathrm{km})$ is much smaller than ours $(\sim 4,000,000 \mathrm{~km})$, which extends into 

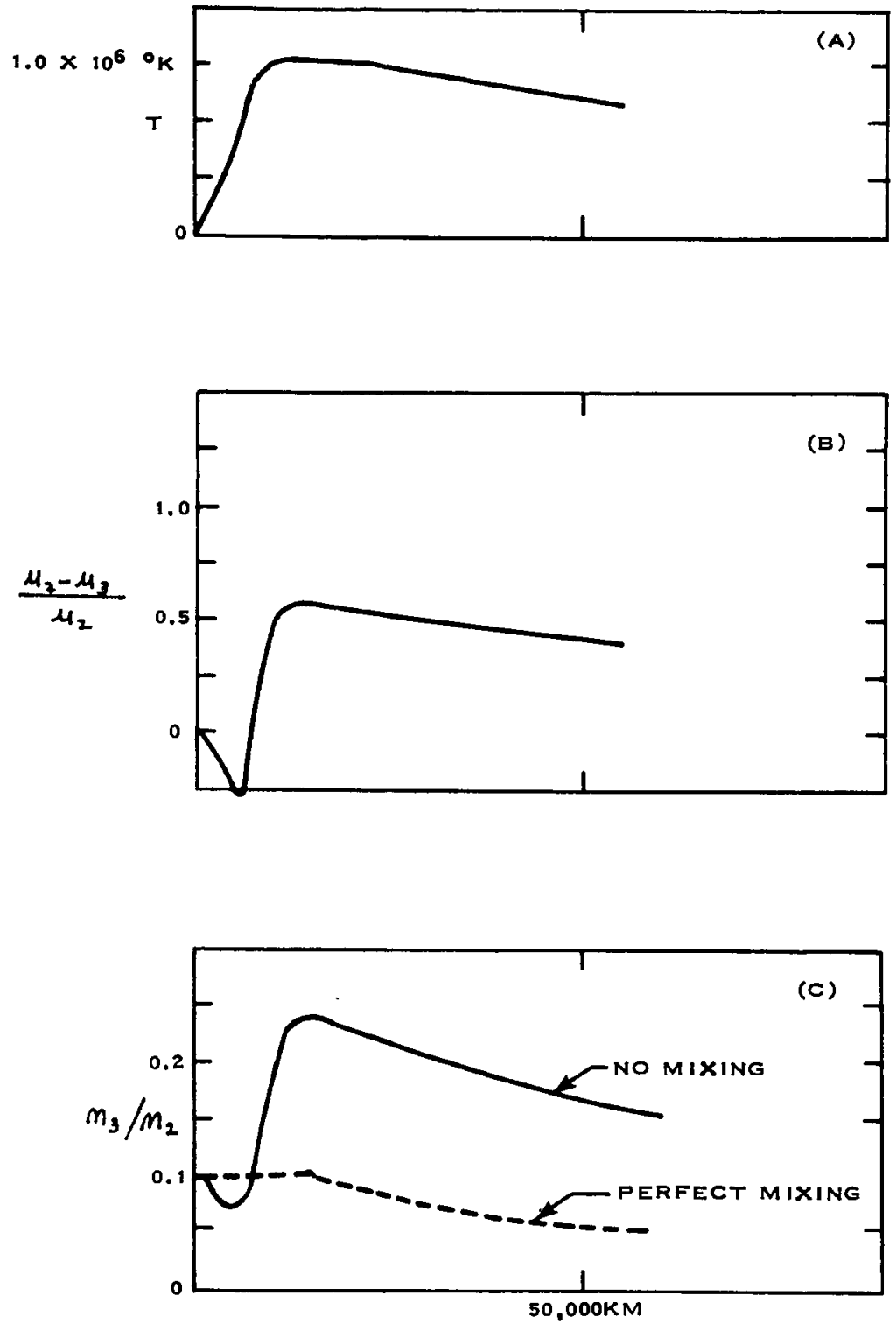

$$
r-R_{\odot}
$$

(A) RADIAL TEMPERATURE VARIATION ASSUMED FOR THE CALCULATIONS

(B) CALCULATED RELATIVE DIFFUSION VELOCITY AS A FUNCTION OF HEIGHT ABOVE THE PHOTOSPHERE

(C) CALCULATED RELATIVE ALPHA-PARTICLE ABUNDANCE AS A FUNCTION OF HEIGHT ABOVE THE PHOTOSPHERE

Figure II. Results of Jokipii for the Diffusion of Alpha Particles in the Chromosphere 
the solar wind proper. To a certain extent then, his results may be regarded as being complementary to those presented in this thesis: the former describe the diffusion of heavy ions through a small region of the lower solar atmosphere with a high degree of detail, the latter describe the diffusion of heavy ions through a much larger region of the solar atmosphere without attempting to investigate the rapid changes taking place as the ions traverse the chromosphere.

Although the results of Jokipii may be regarded as describing correctly the qualitative dynamical behavior of the alpha particles in the chromosphere, it is felt that the treatment offered in this thesis leads to a more accurate description of the diffusion of heavy ions in the solar atmosphere. This follows from the following considerations:

(1) As it was recognized by Jokipii himself, the validity of the diffusion equation of ordinary fluid mechanics that he used is questionable, especially in the outer corona and solar wind, where the mean free paths become very large. (For instance, at $\pi \sim 2.5 \boldsymbol{\Omega}_{\bullet}$, the electron-proton m. f. p. $\sim 3 \times 10^{5} \mathrm{~km}$ ). One specific limitation of that equation is that it is linear in the quantity $\left(\mu_{2}-\mu_{3}\right)$; it will be shown in Chapter II that this linear term is only the leading term of an infinite power series in $\left(\boldsymbol{\mu}_{\mathbf{2}}-\boldsymbol{\mu}_{3}\right)$. The linearized equation will be in error unless the following condition is satisfied:

$$
\left(\frac{\mu_{2}-\mu_{3}}{\mu_{2}}\right) \ll 1
$$


It will later be shown that, contrary to one of Jokipii's assumptions (who stated that, as $r \rightarrow \infty, \mu_{3} \rightarrow \mu_{2}$ ), the quantity $\left(\mu_{2}-\mu_{3}\right.$ ) tends to increase continuously with increasing $\pi$.

(2) Another serious limitation of Jokipii's work lies in his neglect of the effect of the radial electric field due to gravity induced charge separation between the electrons and protons on the heavy ions. It will later be shown that this assumption is not even approximatley valid in the lower chromosphere, and breaks down completely as $r$ increases.

(3) It will later be shown in Chapter III that no single mathematical model is adequate to represent the dynamical and thermal behavior of the corona completely; Jokipii used only one such model, including the simplifying assumption that $T_{1}=T_{2}=T_{3}=T$ everywhere.

(4) Finally, Jokippi presented results for the case of alpha particles only, and did not include calculations for other heavy ions of astrophysical interest. 


\section{DERIVATION OF THE EQUATION OF MOTION FOR THE HEAVY IONS}

Two forms of the radial equation of motion describing the path followed by heavy ions in the solar corona will be derived in this chapter: the first will apply to the case of cool ions which have not yet reached thermal equilibrium with the hot background. The second will apply to the case of ions which have reached thermal equilibrium with the background. Both forms will be sufficiently general to be used with many theoretical models of the coronal background; the only restriction here lies in the assumption that the coronal electrons and protrons may be described by Maxwellian distribution functions.

The derivation itself, although lengthy, is straightforward and follows a basic pattern already given in many standard textbooks, such as "Plasma Kinetic Theory, " by Montgomery and Tidman (1964). The basic starting point is taken to be the Boltzmann kinetic equation for $f_{3}$, the distribution function of the heavy ions:

$$
\frac{\partial f_{3}}{\partial t}+\vec{v} \cdot \frac{\partial f_{3}}{\partial \vec{r}}+\frac{\overrightarrow{F_{3}}}{M_{3}} \cdot \frac{\partial f_{3}}{\partial \vec{v}}=\left(\frac{\delta f_{3}}{\delta t}\right)_{C_{011}}
$$

where $\vec{r}$ and $\vec{v}$ represent the location and velocity of any ion of mass $M_{3}$ in space, $\vec{F}_{3}$ is the applied background force (so far unspecified) on the ion, and $\left(\frac{\delta f_{3}}{\delta t}\right)_{C_{\text {oll }}}$ represents the time rate of change of $f_{3}$ due to 
collisions of the ions with the electrons and protons. The reader will be reminded at this point that we are only interested in the steady state flow motion of the ions in this thesis: all partial time derivatives, such as $\frac{\partial f_{3}}{\partial t}$, will therefore be dropped from now on.

The next step consists in taking zeroth and first order velocity moments of the kinetic equation given by (2), that is to multiply it by $m_{03}$ and $m_{03} M_{3} \vec{N}$ respectively, and to integrate the resulting equations over the velocity space. The results may be written down in onedimensional scalar form directly, since it has already been agreed to assume that the ions and ionized hydrogens were both flowing radially from the sun.

The zeroth order moment equation yields the equation of conservation of particles (conservation of mass), which, in a system exhibiting spherical symmetry, may be written in the following form:

$$
m_{3} \mu_{3} r^{2}=\text { constant }
$$

The first order moment equation yields the equation of conservation of momentum in the radial direction:

$$
u_{3} \frac{d \mu_{3}}{d r}=\frac{\left[\vec{F}_{3}\right]_{\Omega}}{M_{3}}-\frac{1}{M_{3} m_{3}}\left[\frac{d}{d \vec{r}} \cdot \overrightarrow{\vec{P}_{3}}\right]_{\Omega}+\frac{M_{03}}{m_{3}}\left[\int \vec{v}\left(\frac{\delta f_{3}}{\delta t}\right)_{\left.C_{0} 1\right]} d \vec{v}\right]_{\Omega}
$$


where

$$
\overrightarrow{\vec{P}}_{3}=M_{3} M_{03} \int\left(\vec{v}-\vec{\mu}_{3}\right)\left(\vec{v}-\vec{\mu}_{3}\right)_{f_{3}} d \vec{v}
$$

is the pressure tensor, $\vec{\mu}_{3}$ and $M_{3}$ are respectively the flow velocity and the number density of the heavy ions, and the subscript $r$ denotes the radial component of the appropriate vector.

The equation of motion for the heavy ions, (4), is still, however, far from being in a useful form for investigating solutions to the problem; the next step therefore consists in evaluating explicit formulas for the terms $\left[\vec{F}_{3}\right]_{\Omega},\left[\frac{\partial}{\partial \vec{r}} \cdot \overrightarrow{\vec{P}}_{3}\right]_{\Omega}$ and $\left[\int \vec{v}\left(\frac{\delta f_{3}}{\delta t}\right)_{C_{0} I I} d \vec{v}\right]_{\Omega}$

(1) Evaluation of $\left[\vec{F}_{3}\right]_{\Omega}$

A heavy ion, considered as a test particle in the spherically symmetric solar atmosphere will, in general, be subjected to a gravitational, an electric, and a magnetic force:

$$
\vec{F}_{3}=-M_{3} g(\Omega) t+e_{3} \vec{E}(\Omega)+\frac{\ell_{3}}{c}[\vec{v} \times \vec{B}(\Omega)]
$$

where $t$ is a unit vector in the radial direction, and $g(\Omega), \vec{E}(\Omega)$, and $\overrightarrow{\mathbf{B}}(\Omega)$ are the local values of the acceleration of gravity, the electric field, and the magnetic field. 
It should be explained at this point that the "electric field $\vec{E}(r)$ " referred to above is really only one of the two components of the total instantaneous electric field acting on the test particle; it represents the effect of the overall gravity induced charge polarization of the ionized hydrogen background. The other component represents the collective collisional effect of a much smaller group of particles enclosed within a Debye sphere surrounding the test particle; the contribution of this second component will be evaluated separately later when evaluating the collision integral $\left[\int \vec{v}\left(\frac{\delta f_{3}}{\delta t}\right)_{\text {Coll }} d \vec{v}\right]_{\Omega}$

\section{(i) Evaluation of $g(r)$}

Strictly speaking, the term $g(\Omega)$ represents the resultant gravitational interaction between the test particle and all the other particles in the universe. However, it is a well known result of elementary potential theory that, in a system exhibiting spherical symmetry, the gravitational force on a test particle located at a distance $r$ from the center of symmetry will only represent the effect of the particles contained within an imaginary sphere of radius $r$ centered at the origin of the system, since the contribution of the particles located outside that sphere will cancel out identically. In addition, it may be shown 
(see Appendix I) that the contribution of the particles in the solar atmosphere, in the region $r>\Omega_{\bullet}$, on the local value of $g(\Omega)$, is negligible, compared to the contribution of the particles forming the central solar core, in the region $\pi \leqslant \Omega_{0}$. Therefore, to a very good approximation,

$$
g(r)=\frac{G M_{0}}{r_{0}^{2}}\left(\frac{r_{0}}{r}\right)^{2}=g\left(r_{0}\right)\left(\frac{r_{0}}{r}\right)^{2}
$$

where $G$ is the universal gravitation constant and $M_{\odot}$ is the mass of the central solar core.

\section{(ii) Evaluation of $\vec{E}(r)$}

The purpose of this section is to show that in an expanding, fully ionized hydrogen corona, there will exist a radial electric field $E(\Omega)=[\vec{E}(\Omega)]_{\Omega} \quad$ directed away from the sun, and given by the following expression:

$$
E(r)=\frac{M_{2} g\left(\pi_{0}\right)}{l}\left[\frac{1+\frac{c_{0}}{g(r)} \frac{d c_{0}}{d r}}{1+T_{2} / T_{1}}\right]\left(\frac{r_{0}}{r}\right)^{2}
$$

where $M_{2}$ is the mass of a proton, $e$ the unit electrical charge, $c_{0}$ the flow velocity of the corona, and $T_{1}$ and $T_{2}$ the temperatures of the electrons and protons respectively. The physical effect of this electric field on the ions will be to reduce the effective gravitational force acting on them. 
The derivation of the above formula will be given in two steps: a simpler formula will first be obtained, giving the electric field, as sociated with a static ionized hydrogen atmosphere. This first formula will then be generalized to the case of an expanding, accelerating atmosphere. A number of authors have already presented theoretical treatments describing the state of a static, fully ionized, hydrogen stellar atmosphere, including Pannekoek (1922), Rosseland (1924), Van de Hulst (1953), Parker (1963), and Nakada (Unpublished Note). The discussion presented here follows closely the approach taken by Rosseland.

We consider the state of fully ionized matter in a non-rotating (spherically symmetric) star which is in isothermal hydrostatic equilibrium, and, more specifically, the radial distribution of electrons and protons in the star. Because of the force of self-gravitation, we may expect to observe a larger concentration of protons than electrons near the center of the star, and a larger concentration of electrons than protons far away from the center; this will lead to the setting up of a radial electric field $E(r)$ which will counteract the effect of gravity and maintain a stable equilibrium.

We therefore assume that, outside of a spherical shell of radius $r$ drawn around the center of the star there are $N_{e}$ electrons whose 
protons are within so that the inner space bounded by that spherical shell has a net positive charge $\mathrm{N}_{e} e$ and the outer space has a net negative charge $-\mathrm{N}_{e} e$. (See Figure III.)

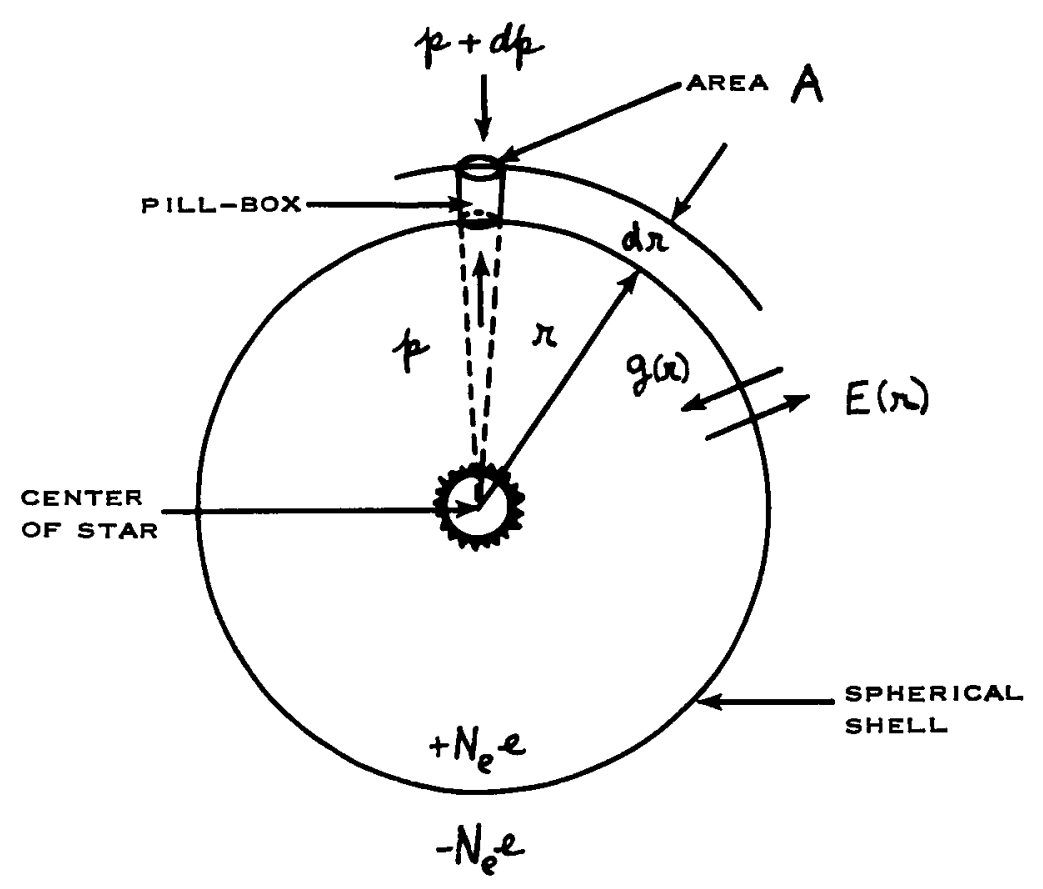

Figure III. Theoretical Model of a Fully Ionized, Spherically Symmetric, Static Stellar A tmosphere

We now draw an imaginary pill-box of base area $A$ between the radii $\pi$ and $\Omega+d \boldsymbol{c}$ (see Figure III) and write the basic equilibrium equations for the electrons and protons contained inside the pill-box 
under the combined effect of the gravitational, pressure, and electrical forces:

where:

$$
\begin{aligned}
& \frac{d p_{1}}{d r}=-p_{1} g-E \sigma_{1} \\
& \frac{d p_{2}}{d r}=-p_{2} g+E \sigma_{2}
\end{aligned}
$$

$$
\begin{aligned}
& p_{1}=\text { partial pressure of the electrons } \\
& p_{2}=\text { partial pressure of the protons } \\
& \rho_{1}=\text { mass density of the electrons } \\
& \rho_{2}=\text { mass density of the protrons } \\
& \sigma_{1}=m_{1} e=\text { magnitude of the charge density of the electrons } \\
& \sigma_{2}=m_{2} e=\text { magnitude of the charge density of the protrons }
\end{aligned}
$$

and the symbols $E=E(r)$ and $g=g(r)$ have been defined already.

The primary purpose of this discussion is to derive an expression for $E=E(x)$ only; however, since $p_{1}, p_{2}, \rho_{1}, \rho_{2}, \sigma_{1}$ and $\sigma_{2}$ are also unknown variables and are directly coupled to $E$, we must simultaneously obtain solutions for these quantities also. We therefore need to set up a system of seven independent equations in these variables, including equations (7) and (8) given already.

The following relations may be written down immediately from the definitions of $\sigma_{1}, \sigma_{2}, \rho_{1}, \rho_{2}$ and from the assumption that the 
electron and proton gases obey the perfect gas law, as if they were dilute gases of electrically neutral point molecules*:

$$
\begin{aligned}
& \sigma_{1}=\frac{\rho_{1} e}{M_{1}} \\
& \sigma_{2}=\frac{\rho_{2} e}{M_{2}} \\
& p_{1}=\frac{\rho_{1} R_{1} T}{M_{1}} \\
& p_{2}=\frac{\rho_{2} R_{1} T}{M_{2}}
\end{aligned}
$$

where $\mathbb{R}$ is the universal gas constant. It has been assumed at this time that $T_{1}=T_{2}=T$; a more general formulation will later be given to include the case $T_{1} \neq T_{2}$.

Finally, a direct application of the integral form of Gauss's law to the spherical shell of radius $\pi$ around the center of the star gives

$$
E=\frac{N_{e} e}{r^{2}}
$$

* This assumption (commonly made in Astrophysics) was shown to be reasonable by Rosseland (1924). 
The solution of equations (7), (8), ... (13) proceeds in the following way: the expressions for $\sigma_{1}$, from (9), $\sigma_{2}$ from (10), $\rho_{1}$ from (11), $\rho_{2}$ from (12), and $\boldsymbol{E}$ from (13) are first substituted into (7), (8) to give:

$$
\begin{aligned}
& \frac{d p_{1}}{d r}=-\frac{p_{1}}{R_{1} T}\left(g \mu_{1}+r\right) \\
& \frac{d p_{2}}{d r}=-\frac{p_{2}}{R_{r} T}\left(g \mu_{2}-r\right)
\end{aligned}
$$

where we have introduced

$$
r=\frac{N_{e} e}{r^{2}}\left(\frac{e}{M_{4}}\right)
$$

Equations ( $\left.7^{\prime}\right)$ and $\left(8^{\prime}\right)$ may now be integrated exactly, provided we limit our attention to the lower regions of the stellar atmosphere where, to a good approximation, $\frac{N_{e}}{\Omega^{2}} \simeq$ constant so that $\gamma$ is a constant.

This gives the following results for $p_{1}, p_{2}, \rho_{1}, \rho_{2}, m_{1}$ and $m_{2}$ :

$$
\begin{aligned}
& p_{1}=p_{1}\left(r_{0}\right) e^{-\frac{\left(r-r_{0}\right)\left(g \mu_{1}+r\right)}{R_{r} T}} \\
& p_{2}=p_{2}\left(r_{0}\right) e^{-\frac{\left(r-r_{0}\right)\left(g \mu_{1}-r\right)}{R_{r} T}} \\
& \rho_{1}=\rho_{1}\left(r_{0}\right) e^{-\frac{\left(r-r_{0}\right)\left(g \mu_{1}+r\right)}{R_{r} T}}
\end{aligned}
$$




$$
\begin{aligned}
& \rho_{2}=\rho_{2}\left(r_{0}\right) e^{-\frac{\left(r-r_{0}\right)\left(g \mu_{2}-r\right)}{R_{1} T}} \\
& m_{1}=m_{1}\left(r_{0}\right) e^{-\frac{\left(r-r_{0}\right)\left(g \mu_{1}+r\right)}{R T}} \\
& m_{2}=m_{2}\left(r_{0}\right) e^{-\frac{\left(r-r_{0}\right)\left(g \mu_{2}-r\right)}{R T}}
\end{aligned}
$$

where $p_{1}\left(n_{0}\right), p_{2}\left(r_{0}\right), \ldots m_{2}\left(n_{0}\right)$ are the values of $p_{1}, p_{2}, \ldots m_{2}$ at the reference level $r=r_{0}$. For $\gamma=0$, the above formulas reduce to the conventional isothermal exponential decay laws for gases consisting of electrically neutral molecules. Incidentally, the above results may also be obtained directly from Boltzmann's principle; however, they are then most commonly written down in the following form, as a function of the electrostatic potential $\Psi$ and the gravitational potential $\Phi$ in the stellar atmosphere: $\quad-\frac{\left(M_{l} \Phi-\ell \Psi\right)}{k T}$

$$
\begin{aligned}
& m_{1}=m_{1}\left(r_{0}\right) e \\
& m_{2}=m_{2}\left(r_{0}\right) e^{-\frac{\left(M_{2} \Phi+e \Phi\right)}{k T}}
\end{aligned}
$$

The required expression for the radial electric field $\boldsymbol{E}(\boldsymbol{r})$ may now be obtained, as was first shown by Rosseland, by noting the close 
mathematical analogy which exists between the electrostatic and the gravitational potentials: $\Psi$ and $\Phi$ may both be obtained from different forms of the Poisson equation:

$$
\begin{aligned}
& \nabla^{2} \Phi=-4 \pi \sigma \\
& \nabla^{2} \Phi=4 \pi G \rho
\end{aligned}
$$

where $\sigma$ (total electric charge density) and $\rho$ (total mass density) may be obtained from $\sigma_{1}, \sigma_{2}, \rho_{1}$ and $\rho_{2}$, for which expressions have been derived already. Therefore,

$$
\begin{aligned}
& \sigma=e\left(m_{2}-M_{1}\right)=e\left[m_{2}\left(r_{0}\right) e^{-\frac{\left(M_{2} \Phi+e \Psi\right)}{k T}}-M_{1}\left(r_{0}\right) e^{-\frac{\left(M_{1} \Phi-e \Psi\right)}{k T}}\right] \\
& \rho=M_{1} M_{1}+M_{2} M_{2}=M_{1} M_{1}\left(r_{0}\right) e^{-\frac{\left(M_{1} \Phi-e \Phi\right)}{k T}}+M_{2} M_{2}\left(r_{0}\right) e^{-\frac{\left(M_{2} \Phi+e \Phi\right)}{k T}}
\end{aligned}
$$

The mathematical form of equations (14), (15), (16) and (17) for $\sigma, \rho, \Psi$ and $\Phi$ suggests, as a trial solution, a linear relation between $\Psi$ and $\Phi$ :

$$
\Psi=K \Phi
$$

where $K$ is so far an undetermined constant. Substitution of this trial solution in the above equations leads to the following result:

$$
\Psi=-\left(\frac{M_{2}-M_{1}}{2 e}\right) \Phi
$$

The radial electric field may now be obtained by taking the gradient of $\Psi$, after substituting the known expression for the gravitational potential $\Phi$ and making the assumption $M_{1} \ll M_{2}$ :

$$
E(\Omega)=\nabla_{\Omega} \Psi \simeq \nabla_{\Omega}\left[\frac{-M_{2} g\left(r_{0}\right) r_{0}^{2}}{2 e r}\right]=\frac{M_{2} g\left(r_{0}\right)}{2 e}\left(\frac{\pi_{0}}{\Omega}\right)^{2}
$$


The reader should be reminded that the above answer only applies to the special case $T_{1}=T_{2}=T$. A more general formula applicable to the case $T_{1} \neq T_{2}$ may easily be derived however, using exactly the same approach as the one outlined above; the resulting electric field will be given by

$$
E(r)=\frac{M_{2} g\left(r_{0}\right)}{e\left(1+T_{2} / T_{1}\right)}\left(\frac{r_{0}}{\Omega}\right)^{2}
$$

This last result is, however, still limited to the case of a static, non-expanding, atmosphere; in addition, it presupposes that $T_{1}$ and $T_{2}$ are constant, since the integration of equations ( $\left.7^{\prime}\right)$ and ( $\left.8^{\prime}\right)$ was directly affected by this. The first of these restrictions may be removed, and the previous formula generalized to the case of an ionized hydrogen atmosphere expanding at a given flow velocity $c_{0}=c_{0}(\Omega)$ by adding, to the gravitational acceleration $g(\Omega)=g\left(r_{0}\right)\left(\frac{\Omega_{0}}{\Omega}\right)^{2}$, the convective acceleration $c_{0} \frac{d c_{0}}{d r}$ experienced by the particles. This is a direct application of Einstein's principle of equivalence. The second restriction may only be removed if explicit formulas are given for $T_{1}=T_{1}(\boldsymbol{r})$ and $T_{2}=T_{2}(\Omega)$ so that the integration of equations $\left(7^{\prime}\right)$ and $\left(8^{\prime}\right)$ may be carried out in closed form.

Strictly speaking, therefore, no truly general formula applicable to all theoretical models of the corona may be given. However, it will 
later be shown in Chapter III that, to a very good approximation, $T_{1}$ and $T_{2}$ do not change very much with $\Omega$ in the integration region considered in this thesis, $\pi_{0} \leqslant \Omega \leqslant 5 \pi_{0}$, whether the model of Parker or the model of Sturrock and Hartle is used. It will therefore be assumed that the following formula for $E(r)$ is sufficiently accurate for our purpose:

$$
E(r)=\frac{M_{2} g\left(r_{0}\right)}{e}\left(\frac{r_{0}}{r}\right)^{2}\left[\frac{1}{1+T_{2} / T_{1}}\right]\left[1+\left(c_{0} \frac{d c_{0}}{d r}\right) \frac{1}{g\left(r_{0}\right)}\left(\frac{r}{n_{0}}\right)^{2}\right]
$$

Near the bottom of the corona, it will later be shown that

$$
c_{0} \frac{d c_{0}}{d r} \ll g\left(r_{0}\right)\left(\frac{r_{0}}{r}\right)^{2}
$$

so that the above formula reduces to the expression obtained previously for the radial electric field in a static atmosphere. Farther away however, the convective term becomes dominant.

\section{(iii) Evaluation of $[\vec{v} \times \vec{B}]_{\Omega}$}

It will be seen in Chapter III that the two theoretical models of the corona considered in this thesis assume that the coronal magnetic field lines are radial and follow the motion of the background fluid. Since the heavy ions are also assumed to flow in the radial direction, the coronal magnetic field $\vec{B}(\Omega)$ will exert no force on them in the direction of motion:

$$
[\vec{v} \times \vec{B}]_{\Omega}=0
$$


We may therefore summarize the previous results by rearranging $\left[\vec{F}_{3}\right]_{\Omega}$ in the following way:

$$
\begin{aligned}
{\left[\vec{F}_{3}\right]_{\Omega} } & =-M_{3} g\left(r_{0}\right)\left(\frac{r_{0}}{\Omega}\right)^{2}+\left(\frac{e_{3}}{e^{\prime}}\right) M_{2} g\left(r_{0}\right)\left(\frac{r_{0}}{r}\right)^{2}\left[\frac{1}{1+T_{2} / T_{1}}\right]\left[1+\frac{c_{0}}{g(\Omega)} \frac{d c_{0}}{d r}\right] \\
& =-M_{3} g\left(r_{0}\right)\left(\frac{r_{0}}{\Omega}\right)^{2}\left\{1-\left(\frac{e_{3}}{e}\right) \frac{M_{2}}{M_{3}}\left[\frac{1}{1+T_{2} / T_{1}}\right]\left[1+\frac{c_{0}}{g(\lambda)} \frac{d c_{0}}{d r}\right]\right\} \\
& =-\eta M_{3} g\left(r_{0}\right)\left(\frac{r_{0}}{r}\right)^{2}
\end{aligned}
$$

In all cases of interest applicable to solar physics considered in this thesis, $0<\eta<1$ : the gravitational force acting on a heavy ion will therefore be reduced by the ionized hydrogen background, but will not be eliminated completely. The following typical values may be given, applicable to the case of an alpha particle $\left(\frac{\ell_{3}}{\ell}=2, \frac{M_{2}}{M_{3}}=\frac{1}{4}\right)$ placed near the base of the corona, where $c_{0} \frac{d c_{0}}{d r} \ll g(r)$ :

$$
\begin{aligned}
& \eta \simeq 3 / 4, \text { if } T_{1}=T_{2} \text { (Parker) } \\
& \eta \simeq 1 / 2, \text { if } T_{1} \gg T_{2} \text { (Sturrock and Hartle) }
\end{aligned}
$$

On the other hand, it is theoretically possible for $\eta$ to be negative: the ion would then "fall" upward faster than the background. A typical example of this phenomenon, given by Oke (1961), would be a hypothetical star whose center atmosphere is composed predominantly of helium: a proton, diffusing out to a radial distance beyond which it 
makes no more collisions with the background, would be projected into outer space with a velocity increasing outward to 0.58 times its gravitational escape velocity from the level at which it was released. This problem was also studied by Nakada (Unpublished Note), who proposed a theory whereby the solar corona would consist predominantly of alpha particles and other heavy ions.

(2) Evaluation of $\left[\frac{\partial}{\partial \vec{x}} \cdot \overrightarrow{\vec{P}}_{3}\right]_{\lambda}$

It has just been shown that $\left[\vec{F}_{3}\right]_{\Omega}$, although a function of $T_{1}$ and $T_{2}$, was independent of the temperature $T_{3}$ of the heavy ions; it will now be seen that $\left[\frac{\partial}{\partial \vec{r}} \cdot \overrightarrow{\vec{P}_{3}}\right]_{\Omega}$ and $\left.\left[\int \vec{N}\left(\frac{\delta f_{3}}{\delta t}\right) d \vec{v}\right]_{\text {oll }}\right]_{\Omega}$ depend explicitly on that temperature. These terms will be evaluated for two specific thermal conditions on the ions:

$$
\begin{aligned}
& T_{3}=0 \quad \text { (Before thermal relaxation with the protons) } \\
& T_{3}=T_{2}=T \text { (After thermal relaxation with the protons) }
\end{aligned}
$$

The case $T_{3}=0$ should of course not be taken literally since no atom could ever be ionized at absolute zero; it is simply a mathematical simplification for the fact that, near the lower limit of integration $\left.\left(\Omega \geqslant r_{0}\right), \frac{T_{3}}{T_{2}} \ll\right]$; typical values would, for instance, be $T_{3} \sim 6 \times 10^{3} \mathrm{OK}$, $T_{2} \sim 10^{6} \mathrm{o}$. Under this assumption, then

$$
f_{3}=\frac{M_{3}}{M_{03}} \delta\left(\vec{N}-\vec{\mu}_{3}\right)
$$


since the ions have no random thermal velocity. Therefore,

$$
\begin{aligned}
\vec{P}_{3} & =M_{3} M_{03} \int \vec{w}_{3} \vec{w}_{3} f_{3} d \vec{v} \\
& =M_{3} M_{3} \int\left(\vec{v}-\vec{\mu}_{3}\right)\left(\vec{v}-\vec{\mu}_{3}\right) \delta\left(\vec{v}-\vec{\mu}_{3}\right) d \vec{v} \\
& =0
\end{aligned}
$$

Physically, this means that a gas of particles at absolute zero will not exert any pressure. There will therefore be no force due to pressure gradient in the radial direction in this case:

$$
\left[\frac{\partial}{\partial \vec{r}} \cdot \overrightarrow{\mathrm{P}}_{3}\right]_{\Omega}=0
$$

The second case $\left(T_{3}=T_{2}\right)$ will be treated by assuming that $f_{3}$ may be represented by a Maxwellian:

$$
f_{3}=\frac{M_{3}}{M_{03}}\left(\frac{M_{3}}{2 \pi k T_{2}}\right)^{3 / 2} e^{-M_{3}\left(\vec{v}-\vec{\mu}_{3}\right)^{2} / 2 k T_{2}}
$$

Therefore,

$$
\overrightarrow{\vec{P}}_{3}=M_{3} M_{3}\left(\frac{M_{3}}{2 \pi k T_{2}}\right)^{3 / 2} \int\left(\vec{v}-\vec{\mu}_{3}\right)\left(\vec{v}-\vec{\mu}_{3}\right) e^{-M_{3}\left(\vec{v}-\overrightarrow{\mu_{3}}\right)^{2 / 2 k T_{2}}} d \vec{v}
$$


The integration of this expression is straightforward. It turns out that

$$
\left[\frac{\partial}{\partial \vec{r}} \cdot \overrightarrow{\vec{P}}\right]_{3}=\frac{d\left(m_{3} k T_{2}\right)}{d r}
$$

(3) Evaluation of $\left[\int \vec{v}\left(\frac{\delta f_{3}}{\delta t}\right)_{C_{0} 11} d \vec{v}\right]_{\pi}$

It will be assumed that $\left(\frac{\delta f_{3}}{\delta t}\right)_{C_{0} 11}$ may be expressed by means of the Fokker-Planck equation:

$\left(\frac{\delta f_{3}}{\delta t}\right)_{C_{011}}=m_{0} \Gamma_{3}\left[-\frac{\partial}{\partial \vec{v}} \cdot\left(f_{3} \frac{\partial h_{3}}{\partial \vec{v}}\right)+\frac{1}{2} \frac{\partial^{2}}{\partial \vec{v} \partial \vec{v}}:\left(f_{3} \frac{\partial^{2} g_{3}}{\partial \vec{v} \partial \vec{v}}\right)\right]$

where the coefficients $\Gamma_{3}, h_{3}$ and $g_{3}$ have been given in standard textbooks, such as those of Montgomery and Tidman (1964), or Holt and Haskell (1965). They are

$$
\begin{aligned}
& \Gamma_{3}=\frac{4 \pi e^{4}}{M_{3}^{2}}\left(\frac{e_{3}}{e}\right)^{2} \log \Lambda \\
& h_{3}=\sum_{j=1}^{2}\left(\frac{M_{j}+M_{3}}{M_{j}}\right) \int \frac{d^{3} \vec{v}_{j} f_{j}\left(\vec{v}_{j}\right)}{\left|\vec{v}-\vec{v}_{j}\right|} \\
& g_{3}=\sum_{j=1}^{2} \int d^{3} \vec{v}_{j}\left|\vec{v}-\vec{v}_{j}\right| f_{j}\left(\vec{v}_{j}\right)
\end{aligned}
$$


where

$$
\Lambda=\frac{3}{2}\left(\frac{k T_{1}}{\ell^{2}}\right)^{3 / 2} \frac{1}{\sqrt{2 \pi M}}
$$

The above formulas automatically take into account the shielding of the electric field of each ion by the rearrangement of the electrons and protons around it. This purely local charge polarization (within a Debye sphere of radius equal to $\lambda_{D}=\left(\frac{k T_{1}}{4 \pi m e^{2}}\right)^{1 / 2} *$ will be in addition to the already mentioned gravity induced charge polarization, which will have a continuous effect on the entire solar atmosphere. However, because of the very high electrical conductivity of the corona, the net local deviation from charge neutrality due to these effects will be very small, and we can still as sume than $\boldsymbol{M}_{\mathbf{1}} \simeq \mathbf{M}_{\mathbf{2}} \simeq \mathbf{M}$ in computing $\boldsymbol{\lambda}_{\mathbf{D}}$ and $\Lambda$

The next step in the evaluation of $\left[\int \vec{v}\left(\frac{\delta / 3}{\delta t}\right)_{C_{011}} d \vec{v}\right]_{\Omega}$ consists in substituting the distribution functions $f_{1}$ and $f_{2}$ (which are assumed to be Maxwellian, respectively peaked at the flow velocities $\mu_{1}$ and $\mu_{2}$ ) explicitly into the previous expression for $\left(\frac{\delta f_{3}}{\delta t}\right)_{C_{\mathbf{0} 11}}$ and in performing the required integrations. The detailed calculations, which are lengthy

* Near the outer corona, $T_{1} \sim 1 \times 10^{6}{ }^{\circ} \mathrm{K}, \boldsymbol{M} \sim 10^{6}$ particles $/ \mathrm{cm}^{3}$, and $\boldsymbol{\lambda}_{\boldsymbol{D}} \sim 6.9 \mathrm{~cm}$. Closer to the sun, $\boldsymbol{\lambda}_{\boldsymbol{P}}$ is even smaller. 
but straightforward, are given in Appendix II. The result turns out to be

$$
\begin{aligned}
& \left(\frac{\delta f_{3}}{\delta t}\right)_{\text {Coll }}=m \Gamma_{3}\left[-\frac{\partial}{\partial \vec{N}} \cdot\left\{f_{3} \frac{\partial}{\partial \vec{v}}\left[\left(\frac{M_{1}+M_{3}}{M_{1}}\right) \frac{1}{w_{1}} \phi\left(w_{1} \sqrt{a}\right)+\left(\frac{M_{2}+M_{3}}{M_{2}}\right) \frac{1}{w_{2}} \phi\left(w_{2} \sqrt{t}\right)\right]\right\}\right. \\
& +\frac{1}{2} \frac{\partial^{2}}{\partial \vec{v} \partial \vec{v}}:\left\{f _ { 3 } \frac { \partial ^ { 2 } } { \partial \vec { v } \partial \vec { v } } \left[\frac { 1 } { 4 a w _ { 1 } } \left(4 w_{1} \sqrt{\frac{a}{n}} e^{-a w_{1}^{2}}+2\left(1+2 a w_{1}^{2}\right) \phi\left(w_{1} \sqrt{a}\right)\right.\right.\right. \\
& \left.\left.\left.+\frac{1}{4 b w_{2}}\left(4 w_{2} \sqrt{\frac{b}{\pi}} e^{-b w_{2}^{2}}+2\left(1+2 b w_{2}^{2}\right) \phi\left(w_{2} \sqrt{2}\right)\right)\right]\right\}\right]
\end{aligned}
$$

where we have introduced:

$$
\begin{aligned}
& w_{1}=\left|\vec{v}-\vec{\mu}_{1}\right| \\
& w_{2}=\left|\vec{v}-\vec{\mu}_{2}\right| \\
& a=M_{1} / 2 k T_{1} \\
& b=M_{2} / 2 k T_{2} \\
& \phi(x)=\frac{2}{\sqrt{\pi}} \int_{0}^{*} e^{-y^{2}} d y \quad \text { (Error Integral) }
\end{aligned}
$$

The last expression for $\left(\frac{\delta f_{3}}{\delta t}\right)_{\text {coll }}$ is still quite general, since an explicit form for $f_{3}$ has not been specified yet. It turns out that the collision integral $\left[\int \vec{r}\left(\frac{\delta / 3}{\delta t}\right)_{c_{0} l l}^{d \vec{r}}\right]_{\Omega}$ will have the same value for both forms of $f_{3}$ selected in this thesis.

The collision integral will take the form:

$$
\frac{M_{03}}{M_{3}}\left[\int \vec{v}\left(\frac{\delta f_{3}}{\delta t}\right)_{\text {Col } 3} d \vec{v}\right]_{\Omega}=m r_{3} \frac{\partial}{\partial u_{3}}\left[\left(\frac{M_{1}+M_{3}}{M_{1}}\right)_{\frac{1}{U_{3,1}}} \phi\left(U_{3,1} \sqrt{a}\right)+\left(\frac{M_{2}+M_{3}}{M_{2}}\right) \frac{1}{U_{3,2}} \phi\left(U_{3,2} \sqrt{t}\right)\right]
$$


where

$$
\begin{aligned}
& U_{3,1}=\left|\vec{\mu}_{3}-\vec{\mu}_{1}\right|=\left|\mu_{3}-\mu_{1}\right| \\
& U_{3,2}=\left|\vec{\mu}_{3}-\vec{\mu}_{2}\right|=\left|\mu_{3}-\mu_{2}\right|
\end{aligned}
$$

Summarizing the previous results, we may therefore write the following two forms for the momentum equation (in the radial direction) of the heavy ions:

A. Before Thermal Equilibrium is Reached with the Solar Corona $\left(T_{3}=0\right)$ :

$$
\begin{aligned}
\mu_{3} \frac{d \mu_{3}}{d r}= & -g\left(\pi_{0}\right)\left(\frac{r_{0}}{r}\right)^{2}\left\{1-\left(\frac{\rho_{3}}{l}\right) \frac{M_{2}}{M_{3}}\left[\frac{1}{1+T_{2} / T_{1}}\right]\left[1+\frac{1}{g\left(r_{0}\right)}\left(c_{0} \frac{d c_{0}}{d r}\right)\left(\frac{r}{r_{0}}\right)^{2}\right]\right\} \\
& +M \Gamma_{3} \frac{\partial}{\partial \mu_{3}}\left\{\left(\frac{M_{1}+M_{3}}{M_{1}}\right) \frac{1}{\left|\mu_{3}-\mu_{1}\right|} \phi\left(\left|\mu_{3}-\mu_{1}\right| \sqrt{a}\right)+\left(\frac{M_{2}+M_{3}}{M_{2}}\right) \frac{1}{\left|\mu_{3}-\mu_{2}\right|} \phi\left(\left|\mu_{3}-\mu_{2}\right| \sqrt{l}\right)\right\}
\end{aligned}
$$

B. After Thermal Equilibrium is Reached with the Solar Corona $\left(T_{3}=T_{2}\right) *$ :

$$
\begin{aligned}
\mu_{3} \frac{d \mu_{3}}{d r}= & -g\left(r_{0}\right)\left(\frac{r_{0}}{r}\right)^{2}\left\{1-\left(\frac{e_{3}}{e}\right) \frac{M_{2}}{M_{3}}\left[\frac{1}{1+T_{2} / T_{1}}\right]\left[1+\frac{1}{g\left(r_{0}\right)}\left(c_{0} \frac{d c_{0}}{d r}\right)\left(\frac{r}{r_{0}}\right)^{2}\right]\right\} \\
& -\frac{1}{M_{3} M_{3}} \frac{d}{d r}\left(M_{3} k T_{2}\right) \\
& +M \Gamma_{3} \frac{\partial}{\partial \mu_{3}}\left\{\left(\frac{M_{1}+M_{3}}{M_{1}}\right) \frac{1}{\left|\mu_{3}-\mu_{1}\right|} \phi\left(\left|\mu_{3}-\mu_{1}\right| \sqrt{a}\right)+\left(\frac{M_{2}+M_{3}}{M_{2}}\right) \frac{1}{\left|\mu_{3}-\mu_{2}\right|} \phi\left(\mu_{3}-\mu_{2} \mid \sqrt{l}\right)\right\}
\end{aligned}
$$

* It has already been explained in Chapter I (Approach to the Problem) that the ions will then acquire the temperature of the protons $\left(T_{2}\right)$ rather than the temperature of the electrons $\left(T_{1}\right)$. 
The basic approach used in solving the above momentum equations for $\mu_{3}=\mu_{3}(x)$ will be to transform them into velocity equations by eliminating the number densities $\boldsymbol{M}_{\mathbf{3}}$ and $\boldsymbol{M} \approx \boldsymbol{M}_{\mathbf{1}} \approx \boldsymbol{M}_{\mathbf{2}}$ with the help of the appropriate conservation of mass equations. The quantities $\mu_{1}=\mu_{1}(\Omega), \mu_{2}=\mu_{2}(\Omega), c_{0}=c_{0}(\Omega), T_{1}=T_{1}(\Omega)$, $T_{2}=T_{2}(x), \Gamma_{3}=\Gamma_{3}(r), a=a(r)$, and $b=b(x)$ will then have to be specified explicitly (either in closed form or not) to leave $\mu_{3}=\mu_{3}(\Omega)$ as the only dependent variable; this step will be presented in Chapter III. However, the above equations will still be, in their present form, exceedingly difficult to solve, even numerically, because of the presence of the error integrals $\phi$ 's in the collision terms; the next step will therefore be to transform these exact integro-differential equations into approximate (but tractable) nonlinear first order differential equations by expanding the error integrals on the assumption that

$$
\begin{aligned}
& \left|\mu_{3}-\mu_{1}\right| \sqrt{a} \ll 1 \\
& \left|\mu_{3}-\mu_{2}\right| \sqrt{q} \ll 1
\end{aligned}
$$

This assumption will now be shown to be reasonable by substituting typical numerical values for $\sqrt{a}, \sqrt{b}, \mu_{1}, \mu_{2}$, and $\mu_{3}$. 
In the physical region of the solar atmosphere under study,

$1 \leqslant \frac{\Omega}{\pi_{0}} \leqslant 5, T_{1} \sim T_{2} \sim 1 \times 10^{6}{ }^{\circ} \mathrm{K}, \mu_{1} \sim \mu_{2} \sim c_{0} \leqslant 1 \times 10^{7} \mathrm{~cm} / \mathrm{sec} . *$ Therefore,

$$
\begin{aligned}
& \sqrt{a}=\sqrt{M_{1} / 2 k T_{1}} \simeq 1.82 \times 10^{-9} \mathrm{sec} / \mathrm{cm} \\
& \sqrt{b}=\sqrt{M_{2} / 2 k T_{2}} \simeq 7.78 \times 10^{-8} \mathrm{sec} / \mathrm{cm}
\end{aligned}
$$

It is much more difficult to assign a typical value to $\mu_{3}$, since this velocity is the unknown quantity to be determined; in fact, $\mu_{3}$ may be positive, negative, or zero: the heavy ions may either move upward, downward, or remain at rest relative to the sun. It is however possible to compute lower and upper bounds to the absolute value $\left|\mu_{3}-c_{0}\right|$ :

(i) Lower Bound: in the "collision-dominated" limit, the force of gravity and the electrical force on each heavy ion will be negligible compared to the kinetic friction force between it and the upward moving ionized hydrogen background, and $\boldsymbol{u}_{\mathbf{3}}=c_{\boldsymbol{0}}$, so that

$$
\left|\mu_{3}-c_{0}\right|_{\text {Min }}=0
$$

\footnotetext{
* These figures apply to the coronal model of Parker; if figures applicable to the model of Sturrock and Hartle are used instead, the approximation becomes even better.
} 
(ii) Upper Bound: in the "gravity-dominated" limit, the kinetic friction force and the electrical force on each heavy ion will be negligible compared to the gravitational force on it, and the ion will move downward in free fall (assuming that it can reach a certain height above the base of the corona by means of some other mechanism in the first place, such as a solar flare).

The familiar principle of conservation of energy may be used to compute the absolute value of the maximum velocity reached by the particle in this way, as it falls from the level $\pi=\Omega$, (at rest) to the lower level $r=\Omega_{0}$; this gives

$$
\left|\mu_{3}\left(r_{0}\right)\right|=\left[2 g\left(r_{0}\right) r_{0}\left(1-\frac{\pi_{0}}{\Omega_{1}}\right)\right]^{1 / 2}
$$

Consequently, $\mu_{3}$ will reach its maximum absolute value if $\boldsymbol{r}_{1}=5 \boldsymbol{\Omega}_{0}$, the upper integration limit; substituting the appropriate numbers for $r_{0}$ and $g\left(\Omega_{0}\right)$, we find that

$$
\left|\mu_{3}\left(n_{0}\right)\right|_{\text {Max }} \simeq 1 \times 10^{7} \mathrm{~cm} / \mathrm{sec} \sim \epsilon_{0} \text { Max }
$$

and therefore

$$
\left|\mu_{3}-c_{0}\right|_{M_{2 x}} \sim 2 c_{\cdot_{\operatorname{Max}}} \sim 2 \times 10^{7} \mathrm{~cm} / \mathrm{sec}
$$

It follows from these results that $\left|c_{0}-\mu_{3}\right|_{M_{2 x}} \sqrt{a} \ll 1$, although $\left|c_{0}-\mu_{3}\right|_{\text {Max }} \sqrt{b} \sim 1$. However, it must be realized that the above 
estimates were based on extreme physical conditions which are very unlikely to exist in reality*.

It will therefore be assumed from now on that

$$
\begin{aligned}
& \left|\mu_{3}-\mu_{1}\right| \sqrt{a} \ll \mid \\
& \left|\mu_{3}-\mu_{2}\right| \sqrt{b} \ll 1
\end{aligned}
$$

and this will be checked later in Chapter IV. It is also logical to assume that $\mu_{3}<\mu_{1}, \mu_{3}<\mu_{2}$ since the ions, even if moving upward, should experience some settling due to gravity. This will also be checked later.

We are now able to expand the error integrals (and their derivatives) by means of convergent alternating series of the form

$$
\begin{aligned}
\phi(y) & =\frac{2}{\sqrt{n}} \int_{0}^{y} e^{-x^{2}} d x \\
& =\frac{2}{\sqrt{\pi}} \int_{0}^{y}\left[1-x^{2}+\frac{x^{4}}{2 !}+\ldots+(-1)^{n} \frac{x^{2 n}}{n !}\right] d x \\
& =\frac{2}{\sqrt{n}} \sum_{x=0}^{\infty} \frac{(-1)^{x} y^{2 x+1}}{(2 x+1) x !} \\
\text { where } y & =\left|\mu_{3}-\mu_{1}\right| \sqrt{a} \quad \text { or }\left|\mu_{3}-\mu_{2}\right| \sqrt{b}
\end{aligned}
$$

* For instance, it will later be seen in Chapter IV that the maximum value actually reached by $\left|\mu_{3}-c_{0}\right|$ in our calculations (with HeII as the ion) never exceeded $0.5 c_{0}$; this would then satisfy both assumptions unconditionally. 
The collision term in the right-hand side of the two forms of the equation of motion will then become, after simplification:

$$
\begin{aligned}
& \frac{2 m r_{3}}{\sqrt{\pi}}\left\{\left(\frac{M_{1}+M_{3}}{M_{1}}\right) \sum_{x=1}^{\infty} \frac{(-1)^{x}\left(\mu_{1}-M_{3}\right)^{2 x-1} a^{\frac{2 x+1}{2}}}{x !}\left(\frac{-2 x}{2 x+1}\right)\right. \\
& \left.+\left(\frac{M_{2}+M_{3}}{M_{2}}\right)_{x=1}^{\infty} \frac{(-1)^{x}\left(\mu_{2}-\mu_{3}\right)^{2 x-1} b^{\frac{2 x+1}{2}}}{x !}\left(\frac{-2 x}{2 x+1}\right)\right\}
\end{aligned}
$$

If this is applied to the model of Parker, or to the model of Sturrock and Hartle, $\mu_{1}=\mu_{2}=c_{0}$; the above form of the friction term may then be further simplified to give the following result:

$$
\begin{aligned}
\frac{M_{03}}{M_{3}}\left[\int \vec{v}\left(\frac{\delta f_{3}}{\delta t}\right)_{c_{0} 11} d \vec{v}\right. & = \\
& \frac{16 M_{\Omega} e^{4} \sqrt{n}}{M_{3}^{2}}\left(\frac{e_{3}}{l}\right)^{2} \log \left[\frac{3}{2}\left(\frac{\left.k T_{1}\right)^{3 / 2}}{e^{2}} \frac{1}{\sqrt{2 \pi M}}\right] \sum_{x=1}^{\infty} \frac{(-1)^{x+1}\left(c_{0}-43\right)^{3 x-1}}{(x-1) !(2 x+1)}\left(\frac{M_{2}+M_{3}}{M_{2}}\right) b^{x+\frac{1}{2}}\right.
\end{aligned}
$$

since, in our calculations;

$$
\left(\frac{M_{2}+M_{3}}{M_{2}}\right) b^{X+\frac{1}{2}} \gg\left(\frac{M_{1}+M_{3}}{M_{1}}\right) a^{X+\frac{1}{2}}, X=1,2, \ldots \ldots \infty
$$

and the explicit expression for $\Gamma_{3}$ was substituted.

We may therefore use the same general formula to compute the effect of friction, regardless of which theoretical model is used to describe the background; the only difference will be that the quantities $T_{1}$ and $b$ (by definition, $b=M_{2} / 2 k T_{2}$ ) will be constant in one case, variable in the other. 
The two forms of the equation of motion may now be written down formally by combining together (4), ( 19$),(20),(21)$ and ( 22 ). However, it will later be found useful, in computations leading to solutions of the problem, to work with dimensionless equations. These will be obtained by introducing the following new dimensionless variables $\xi, U, V$, and $r$ :

$$
\begin{aligned}
& \zeta=r / r_{0} \\
& c_{0}=U \sqrt{2 p_{0} / \rho_{0}} \\
& \mu_{3}=V \sqrt{2 p_{0} / \rho_{0}} \\
& m=r m_{0}
\end{aligned}
$$

where $m_{\bullet}, p_{\bullet}$, and $\rho_{\bullet}$ are respectively the values of the total number density, pressure, and mass density of the corona at the reference radius $\Omega_{0}=\pi_{0}$. In both theoretical models of the corona considered in this thesis, the electrons and protons, which make up the bulk of the corona, are assumed to be at the same temperature $T_{1}=T_{2}=T_{0}$ at the radius $\Omega=\Omega$. and to obey the perfect gas low. We may therefore substitute

$$
\frac{p_{0}}{\rho_{0}}=\frac{2 k T_{0}}{M_{2}}
$$

in the previous expressions. This will give the following simplified forms for the dimensionless equations describing the motion of the ions in the corona: 
A. Before Thermal Equilibrium is Reached with the Solar Corona $\left(T_{3}=0\right)$ :

$$
\begin{aligned}
V \frac{d V}{d \zeta}=-\frac{F_{1}}{s^{2}} & +F_{2} U \frac{d u}{d s} \\
& +F_{3} r \log \frac{F_{4}}{\sqrt{r}}\left[\sum_{x=1}^{\infty} \frac{(-1)^{x+1}(2)^{x}(u-V)^{2 x-1}}{(x-1) !(2 x+1)}\left(\frac{T_{0}}{T_{2}}\right)^{x+\frac{1}{2}}\right]
\end{aligned}
$$

B. After Thermal Equilibrium is Reached with the Solar Corona $\left(T_{3}=T_{2}\right)$ :

$$
\begin{aligned}
\frac{V d V}{d \xi}=-\frac{F_{1}}{\xi^{2}} & +F_{2} U \frac{d U}{d \xi}-\frac{F_{5}}{M_{3}} \frac{d}{d \xi}\left(M_{3} T_{2}\right) \\
& +F_{3} r \log \frac{F_{4}}{\sqrt{r}}\left[\sum_{x=1}^{\infty} \frac{(-1)^{x+1}(2)^{x}(U-V)^{2 x-1}}{(x-1) !(2 x+1)}-\left(\frac{T_{0}}{T_{2}}\right)^{x+\frac{1}{2}}\right]
\end{aligned}
$$

where

$$
\begin{aligned}
& F_{1}=r_{0} g\left(r_{0}\right)\left(\frac{M_{2}}{4 k T_{0}}\right)\left[1-\frac{l_{3}}{l}\left(\frac{M_{2}}{M_{3}}\right)\left(\frac{1}{1+T_{2} / T_{1}}\right)\right] \\
& F_{2}=\frac{l_{3}}{l}\left(\frac{M_{2}}{M_{3}}\right)\left(\frac{1}{1+T_{2} / T_{1}}\right) \\
& F_{3}=\frac{2 r_{0} M_{0} e^{4}}{\left(k T_{0}\right)^{2}} \sqrt{\frac{\pi}{2}}\left(\frac{l_{3}}{l}\right)^{2}\left(\frac{M_{2}}{M_{3}}\right)^{2}\left(1+\frac{M_{3}}{M_{2}}\right) \\
& F_{4}=\frac{3}{2}\left(\frac{k T_{0}}{l^{2}}\right)^{3 / 2} \frac{1}{\sqrt{2 \pi M_{0}}}\left(\frac{T_{1}}{T_{0}}\right)^{3 / 2} \\
& F_{5}=\frac{M_{2}}{4 M_{3} T_{0}}
\end{aligned}
$$


It should be noted that, for a given type of ions, and for given boundary conditions at $\boldsymbol{r}=\boldsymbol{r}_{0}, \boldsymbol{F}_{\mathbf{3}}$ and $\boldsymbol{F}_{\mathbf{5}}$ will be known constants; furthermore, if the model of Parker is selected to represent the background, $T_{1}=T_{2}=T_{0}=$ constant, so that $F_{1}, F_{2}$, and $F_{4}$ will also be known constants. If the model of Sturrock and Hartle is selected instead, $T_{1}$ and $T_{2}$ become specified functions of $\Omega$ (or $\xi$ ), so that $F_{1}, F_{2}$, and $F_{4}$ will be given variables. 


\section{THEORETICAL MODELS OF THE SOLAR CORONA}

Before attempting to solve the diffusion equations (23) and (24) for the flow velocity $V$ of the ions in the corona, we must specify, as a function of position, the basic flow properties (dimensionless) of the ionized hydrogen background which appear in these equations: the flow velocity $u$, the total number density $r$, and the temperatures $T_{1}$ and $T_{2}$ of the electrons and protons. As it was previously explained in the second part of Chapter I (Approach to the Problem), these quantities are assumed to be identical to those obtained by other authors for a simplified corona consisting of fully ionized hydrogen only. A brief historical review of the work accomplished in the dynamical study of a corona consisting of ionized hydrogen only will now be given, followed by a more detailed description of the two coronal models specifically selected to solve the diffusion equations (23) and (24).

A model of a thermally conducting, static, corona was first obtained by Chapman (1957), who derived the temperature distribution of such an atmosphere. The concept of a static solar atmosphere was criticized by Parker (1958, 1963), who suggested instead a model in which energy is transferred outward from the base of the corona by hydrodynamic streaming. In this model (described in detail below), 
the fluid is accelerated outward by pressure gradients so that it arrives at the earth with a radial streaming velocity around 400 to $800 \mathrm{~km} / \mathrm{sec}$. The solar wind observed near the earth may therefore be regarded as a manifestation of the expanding corona. Another theory, proposed by Chamberlain (1961), retained the concept of a thermally conducting convective solar atmosphere, but suggested that the corona is heated only in a thin shell at its base. Noble and Scarf (1963) pursued this idea, taking boundary conditions appropriate to the solar wind: these results have tended to confirm the predictions of Parker's model. A viscous model of the solar wind, obtained by solving the Navier - Stokes equations of ordinary fluid mechanics, was also brought forward by Whang, Liu, and Chang (1966).

Even though fully ionized hydrogen consists of two fluids (electrons and protons), all the theoretical models of the corona mentioned above assume that the electrons and protons have the same temperature, the same flow velocity, and the same local number density: they may therefore be described as being "one-fluid" models. It will later be shown that typical values of $r_{\boldsymbol{E}}$, the collision frequency for energy exchange between electrons and protons, are such that one should expect the electron and proton temperatures to be quite different in the solar wind. Such a "two-fluid" model was investigated by Sturrock and Hartle (1966) and will be described below. 


\section{A. Model of Parker}

This model of the corona and solar wind rests on the fact that the mean free path of the average thermal proton* is short compared to the relevant dynamical lengths, such as the scale height, solar radius, etc. According to Parker then, the corona may be regarded as a hydrodynamic atmosphere rather than a gas of discrete charged particles. It follows from this reasoning that the classical one-fluid

* By definition, the m.f.p. is given by the formula m.f.p. $=\bar{\sim} t_{D}$

$$
\text { where: } \quad \begin{aligned}
\vec{v}= & \text { thermal velocity of proton } \\
t_{\mathbf{D}}= & \text { time in which a proton undergoes an } \\
& \text { accumulative deflection of the order of } \\
& 900 \text { due to coulomb scattering in an at }- \\
& \text { mosphere of ionized hydrogen of } \mathrm{m} \text { ions } / \mathrm{cm}^{3} \\
\simeq & 3 \times 10^{-12} \vec{v}^{3} / \mathrm{Mlog} \wedge \\
= & 3 \times 10^{-12} \overline{\mathrm{v}}^{3} / \mathrm{Mlog}\left[1.24 \times 10^{4} \mathrm{~T}^{\left.3 \mathrm{~h} / \mathrm{m}^{1 / 2}\right]}\right.
\end{aligned}
$$

For typical values of $\boldsymbol{M}$ and $T$, this gives

$$
\begin{aligned}
& \text { m.f.p. } \simeq 3 \times 10^{2} \mathrm{~km} \text { at } \Omega=\Omega \\
& \text { m.f.p. } \simeq 3 \times 10^{4} \mathrm{~km} \text { at } \Omega \simeq 1.3 \Omega \\
& \text { m.f.p. } \simeq 3 \times 10^{5} \mathrm{~km} \text { at } \Omega=1.75 \Omega .
\end{aligned}
$$


hydrodynamic equations may be used to study the overall expansion of the corona, using a hydrostatic pressure which is maintained isotropic by Coulomb scattering.

Making the usual assumption of steady-state motion along the radial direction, and neglecting solar rotation, the equation of motion describing the behavior of the system will be

$$
c_{0} \frac{d c_{0}}{d r}+\frac{1}{\rho} \frac{d p}{d r}+\frac{d \Phi}{d r}=0
$$

where $c_{0}$ is the flow velocity, $\rho$ the gas density, and $\Phi=-G M_{\odot} / r$ is the gravitational potential. The force exerted on the gas by the magnetic field $\overrightarrow{\mathbf{B}}$ has no component along the radial direction, since $\overrightarrow{\mathrm{B}}$ is radial; in addition, no electrical force appears in the above equation, since the postulate that we have a "one-fluid" gas automatically excludes any charge separation effect. Finally, viscous forces have been neglected in (25) because they appear to be rather smaller than the effects of thermal conduction along the streamline; the effects of coronal heating and thermal conduction are represented by supposing that $p$ and $\rho$ are related by a polytrope law:

$$
\left(\frac{p_{p}}{p_{0}}\right)=\left(\frac{p}{\rho_{0}}\right)^{\alpha}
$$


where $\alpha$ is the polytrope index; in general, $1 \leqslant \alpha \leqslant 5 / 3: \alpha=1$ describes an isothermal gas, $\alpha=5 / 3$ describes an adiabatic gas. As a final equation, we may write the equation of conservation of mass:

$$
\rho c_{0} r^{2}=\rho_{0}\left(c_{0}\right)_{0} r_{0}^{2}
$$

It is now possible to solve the system of equations (25), (26), and $(27)$ for the required flow quantities $c_{0}, p$, and $p$. The temperature $T$ may then be obtained from the perfect gas law. However, it is again (as in Chapter II) more convenient to introduce beforehand a dimentionless gravitational potential by defining $H=G M_{0} \rho_{0} / x_{0} p_{0}$, as well as the other dimensionless quantities already defined in Chapter II. The solution for $\boldsymbol{U}$, the dimensionless form of $\boldsymbol{c}_{0}$, cannot be expressed in closed form exactly, but may be obtained by solving transcendental equations:

For $\alpha=1$,

$$
U^{2}-\log U=2 \log \xi+H / S+U_{2}^{2}
$$

For $\alpha \neq 1$,

$$
U^{2}+\frac{\alpha}{\alpha-1}\left(\frac{u_{1}}{u f^{2}}\right)^{\alpha-1}=\frac{H}{2}+U_{1}^{2}
$$

where

$$
\begin{aligned}
& u_{1}^{2}=u_{0}^{2}+\alpha /(\alpha-1)-H \\
& u_{2}^{2}=u_{0}^{2}-\log u_{0}-H
\end{aligned}
$$

Mathematically, the solution of equations (28) and (29) is closely analogous to the expansion of a gas through a Laval nozzle 
and is complicated by the many branches of the solutions of the Bernouilli equation. For simplicity, we will only use, in calculations presented in this thesis, the isothermal results $(\alpha=1)$ : there is in fact no single effective value of $\alpha$ which is valid throughout the entire corona. However, the best fit suggested by Parker consists of taking $\alpha=1$ from $\Omega=\Omega_{0}$ to some transition radius $\pi=\Omega_{2}$, beyond which $\alpha \simeq 5 / 3$. Since the integration range considered in this thesis is $r_{0} \leqslant \Omega \leqslant 5 \Omega_{0}$, the choice $\alpha=1$ seems logical.

We now return to the topology of the Bernouilli equation. A typical set of results for $\alpha=l$ is reproduced from the book of Parker in Figure IV.

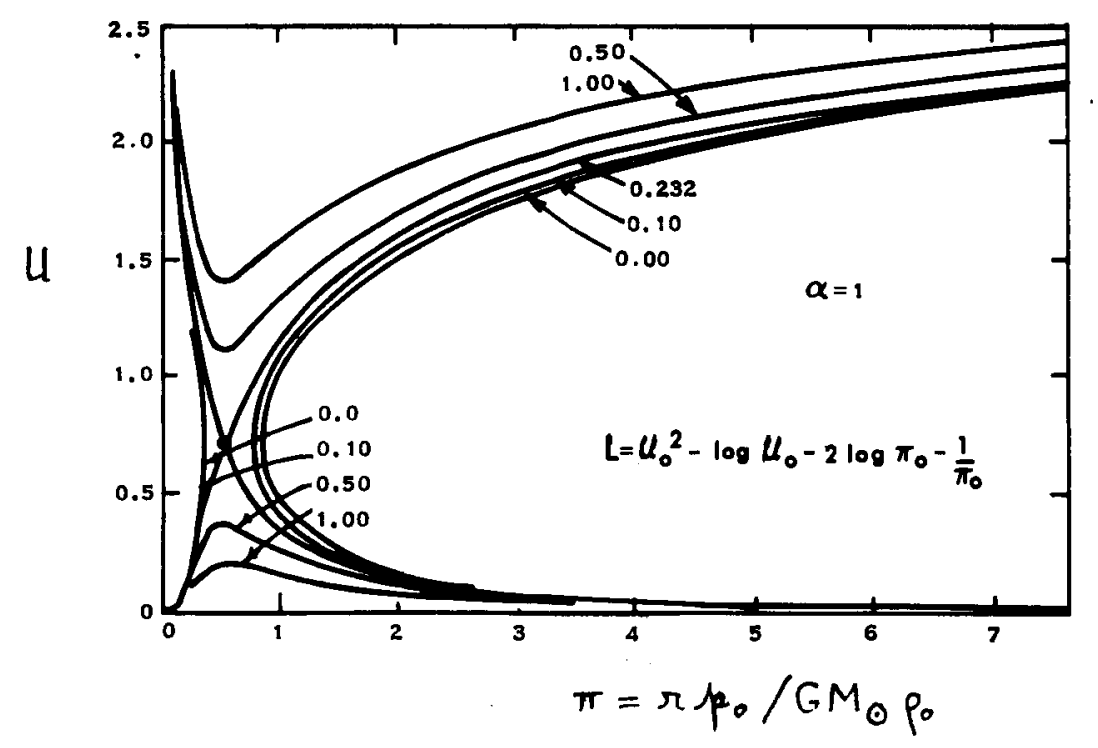

Figure IV. The Family of Solutions of the Bernouilli Equation (28), $U$ versus $\pi$ (or $\pi$ ), for an Isothermal Corona and Various Values of the Constant $L$ Designated on each Curve. The Critical Point, marked by the Large Dot, has the Coordinates $U_{c}=1 / \sqrt{2}, \pi_{c}=1 / 2$. 
It will be noticed that there are two branches of the velocity curve near the sun and two far from the sun. The lower curve is the appropriate one near the sun, since the expansion velocity must go to zero there; the upper curve is the appropriate one far from the sun, giving supersonic velocity and vanishing gas pressure. A physically meaningful solution will cross over from the lower to the upper branch at some point called a critical point; this is similar to the cross-over from subsonic to supersonic flow in a Laval nozzle. It may be shown that, for $\alpha=1$, the critical point is $U_{c}=1 / \sqrt{2}$ and $\zeta_{c}=H / 2$. The value of $U_{2}^{2}$ for the solution through the critical point is

$$
u_{2}^{2}=\frac{1}{2}(\log 2+1)-2\left(\log \frac{H}{2}+1\right)
$$

A number of solutions $c_{0}=c_{0}(r)$ corresponding to different constant temperatures $T_{0}$ was also given in Parker's work and is reproduced in Figure $V$ of this thesis. It will be noticed that $c_{0}$ increases very rapidly with $\Omega$ close to the sun, and more slowly thereafter.

Finally, it should be mentioned that for small values of $\xi$, an approximate asymptotic solution may be written in closed form for $U$ :

$$
u \simeq \frac{1}{\xi^{2}} e^{-\left(\frac{H}{\xi}+U_{2}^{2}\right)}
$$




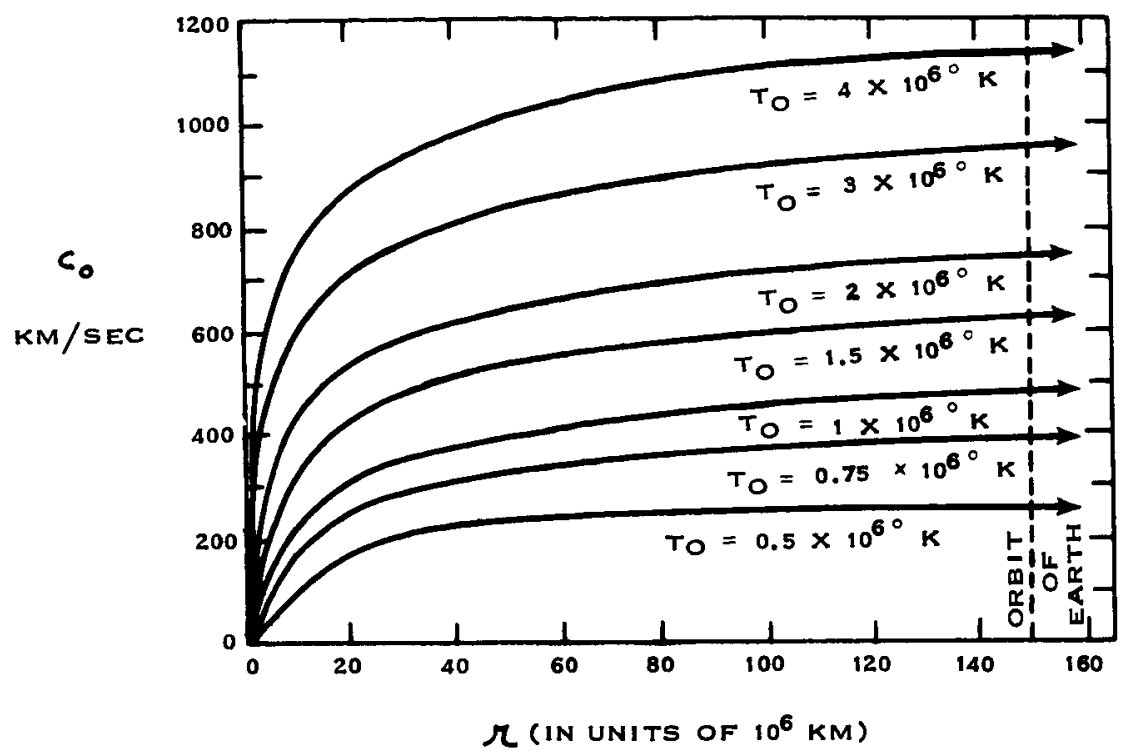

Figure V. Expansion Velocity $c_{0}(\mathrm{~km} / \mathrm{sec})$ of an Isothermal Corona as a Function of the Radial Distance $r$ (in units of $10^{6} \mathrm{~km}$ ) from the center of the Sun. 


\section{B. Model of Sturrock and Hartle}

The primary reason for introducing this "two-fluid" model rests in the fact that the collision frequency $r_{E}$ for energy exchange between the electrons and protons does not seem to be sufficiently high (except very close to the sun) to maintain thermal equilibrium between the se species, so that it may be expected that the temperatures of the electrons and protons could become different in the outer corona and solar wind. The basic formula for $r_{\mathbf{E}}$ was given by Spitzer (1962):

$$
r_{E}=8.5 \times 10^{-2} \mathrm{mT}^{-3 / 2}
$$

This will be compared to the expansion rate $r_{\boldsymbol{e}}=-\frac{\boldsymbol{c}_{0}}{\boldsymbol{m}} \frac{d \boldsymbol{m}}{d \boldsymbol{r}} *$ of the solar atmosphere, using "one-fluid" values for $c_{0}$ and $\boldsymbol{m}$ obtained with the model of Parker described previously in Section A of this chapter**.

\footnotetext{
* Far from the sun, where $c_{\bullet}$ does not vary too rapidly with $\Omega$, the above formula for $r_{e}$ becomes, approximately, $r_{e} \simeq 2 c_{0} / \Omega$

** In their original paper, Sturrock and Hartle (1966) compared $r_{e}$ and $r_{e}$ at only one value of $\Omega\left(\Omega \approx 150 \Omega_{0}\right)$, where $r_{E} \ll r_{e}$; they used, in their calculations, the "one-fluid" model of Scarf and Noble (1965) instead of the isothermal model of Parker (1963) used in this thesis.
} 
In order to be consistent with the rest of the treatment, only the isothermal Parker model $(\alpha=1)$ will be considered. It will later be shown in Chapter IV that no single value of $T$ is then entirely satisfactory; however, most authors consider the temperature of an isothermal corona to lie between $1 \times 10^{6} \mathrm{oK}$ and $2 \times 10^{6} \mathrm{oK}$. Numerical values for $r_{E}$ and $r_{e}$ have been computed for these two temperatures and for three different distances from the sun: $\Omega=\Omega_{0}, \Omega=5 \Omega_{0}$, and $\pi=150 \Omega_{0}$ (radius of earth's orbit around the sun); they are given in Table 1 .

\begin{tabular}{|c||c|c|c|c|c|c|}
\hline$T$ & \multicolumn{3}{|c|}{$1 \times 10^{6} \circ \mathrm{K}$} & \multicolumn{3}{c|}{$2 \times 10^{6} \circ \mathrm{K}$} \\
\hline$\zeta=\Omega / \Omega_{0}$ & 1 & 5 & 150 & 1 & 5 & 150 \\
\hline$r_{E}\left(\sec ^{-1}\right)$ & $2.6 \times 10^{-3}$ & $2.1 \times 10^{-6}$ & $7.4 \times 10^{-10}$ & $9.0 \times 10^{-4}$ & $6.8 \times 10^{-6}$ & $3.5 \times 10^{-9}$ \\
\hline$r_{e}\left(\sec ^{-1}\right)$ & $3.4 \times 10^{-5}$ & $8.9 \times 10^{-5}$ & $6.5 \times 10^{-6}$ & $2.7 \times 10^{-4}$ & $1.7 \times 10^{-4}$ & $9.9 \times 10^{-6}$ \\
\hline
\end{tabular}

Table 1. Typical values of the collision frequency $r_{\boldsymbol{E}}$ and the expansion rate $r_{e}$ in the corona and solar wind.

The following conclusions may be obtained from the results given in Table 1:

(1) Near the bottom of the corona $\left(\Omega / \pi_{a} \sim 1\right), r_{E}>r_{e}$ : thermal equilibrium may then be assumed to exist between the electrons and protons, and $T_{1} \simeq T_{2}$. 
(2) Far away in the solar wind $\left(\pi / r_{0} \sim 150\right), r_{E} \ll r_{e}:$ thermal equilibrium may no longer be assumed to be maintained between the electrons and protons, and $T_{1} \neq T_{2}$.

(3) For intermediate distances, such as the upper limit of integration considered in this thesis $\left(\Omega / \Omega_{0}=5\right)$, no definite answer may be given.

The assumption $T_{1}=T_{2}=T$ made previously by Parker is therefore relaxed. However, the electrons and protons are still assumed to have the same number density $m$, the same flow velocity $c_{0}$, and to behave as perfect gases. The equation of continuity will have the usual form

$$
m c_{0} r^{2}=J
$$

where $J$ is a constant. The dynamical equation will be, if we neglect solar rotation, viscous stresses, non-radial magnetic fields, and $M_{1} \ll M_{2}:$

$$
m M_{2} c_{0} \frac{d c_{0}}{d r}=-\frac{d}{d r}\left(m k T_{1}+m k T_{2}\right)-\frac{G M_{0} m M_{2}}{\Omega^{2}}
$$

The energy equation given by Braginskii (1965) is then combined with equation (31) and (32) given above to obtain "heat equations" for protons and electrons :

$$
\begin{aligned}
& \frac{3}{2} \frac{1}{T_{2}} \frac{d T_{2}}{d r}-\frac{1}{m} \frac{d m}{d r}=\frac{1}{J k T_{2}} \frac{d}{d r}\left(r^{2} X_{2} \frac{d T_{2}}{d r}\right)+\frac{3}{2} \frac{r_{E}}{C_{0}}\left(\frac{T_{1}-T_{2}}{T_{2}}\right) \\
& \frac{3}{2} \frac{1}{T_{1}} \frac{d T_{1}}{d r}-\frac{1}{m} \frac{d M}{d r}=\frac{1}{J k T_{1}} \frac{d}{d r}\left(r^{2} X_{1} \frac{d T_{1}}{d r}\right)+\frac{3}{2} \frac{r_{E}}{C_{0}}\left(\frac{T_{2}-T_{1}}{T_{1}}\right)
\end{aligned}
$$


where $\boldsymbol{K}_{1}$ and $\boldsymbol{K}_{2}$ are respectively the thermal conductivities of the electrons and protons. They may be expressed by means of the following formulas, first given by Chapman (1954):

$$
\begin{aligned}
& K_{1} \simeq 6 \times 10^{-7} \mathrm{~T}_{1}^{5 / 2} \\
& K_{2} \simeq 1.4 \times 10^{-8} \mathrm{~T}_{2}^{5 / 2}
\end{aligned}
$$

In addition,

$$
r_{E} \simeq 8.5 \times 10^{-2} M T_{1}^{-3 / 2}
$$

The above system of equations was solved numerically by Sturrock and Hartle with the boundary conditions that at $r=R_{\odot}$, $M=M_{0}, T_{1}=T_{2}=T_{0}$; they also required that there must be a subsonicsupersonic transition at some point $\Omega>R_{\odot}$. Radius dependent polytrope indices $\alpha_{1}(\Omega)$ and $\alpha_{2}(\Omega)$ were introduced to avoid solving all the differential equations simultaneously, such that

$$
\begin{aligned}
& T_{2}\left(\frac{m}{M_{0}}\right)^{1-\alpha_{2}}=T_{0} \\
& T_{1}\left(\frac{m}{m_{0}}\right)^{1-\alpha_{1}}=T_{0}
\end{aligned}
$$

It turns out that $\alpha_{1}$ and $\alpha_{2}$ may be represented by the following formulas:

$$
\begin{aligned}
& \alpha_{1}=\alpha_{1}(\Omega)=\delta_{1}-\left(\delta_{1}-\beta_{1}\right) e^{-\left(\Omega-R_{0}\right) / H_{1}} \\
& \alpha_{2}=\alpha_{2}(\Omega)=\delta_{2}-\left(\delta_{2}-\beta_{2}\right) e^{-\left(\Omega-R_{0}\right) / H_{2}}
\end{aligned}
$$

where $\delta_{1}, \delta_{2}, \beta_{1}, \beta_{2}, H_{1}$ and $H_{2}$ are constants. 
Only one set of numerical results was obtained by Sturrock and Hartle, corresponding to $T_{0}=2 \times 10^{6} 0 \mathrm{~K}$ and $m_{0}=3 \times 10^{7} / \mathrm{cm}^{3}$.

They are reproduced as Figure VI and Figure VII in this thesis and give $c_{0}=c_{0}(\pi), m=m(\pi), T_{1}=T_{1}(\pi)$, and $T_{2}=T_{2}(\pi)$. The results for $c_{0}$ and $m$ seem to have the same general qualitative behavior than the results of Parker: in the range $1 \leqslant \frac{\pi}{r_{0}} \leqslant 5$, the velocity c. is consistently slightly lower than the corresponding velocity obtained by Parker at the same point for $T_{0}=2 \times 10^{6} \mathrm{~K}$. It should be recognized that the two sets of results are not based on exactly the same boundary conditions, since Sturrock and Hartle used $R_{\odot}$ as their reference radius, and not $\pi_{0}$ as Parker $\left(r_{0} \simeq 1.43 R_{\odot}\right)$.

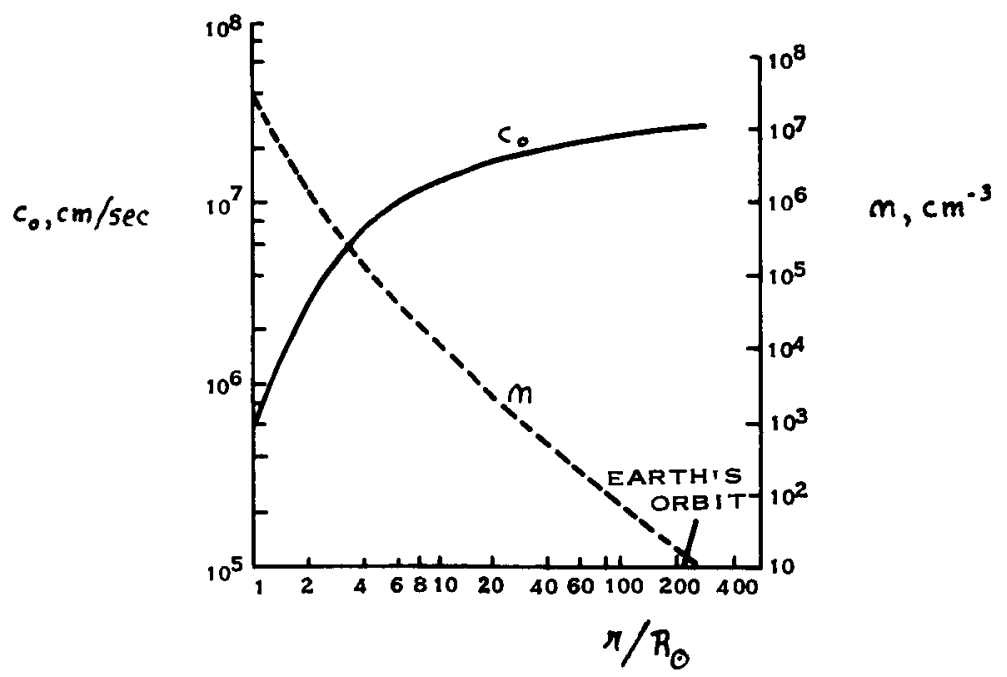

Figure VI. The flow velocity $c_{0}\left(\mathrm{~cm} \mathrm{sec}^{-1}\right.$, solid line) and electron density $m\left(\mathrm{~cm}^{-3}\right.$, broken line) as a function of the radial distance (in units of $R_{\odot} \simeq 7 \times 10^{10}$ $\mathrm{cm}$ ) from the center of the sun. 


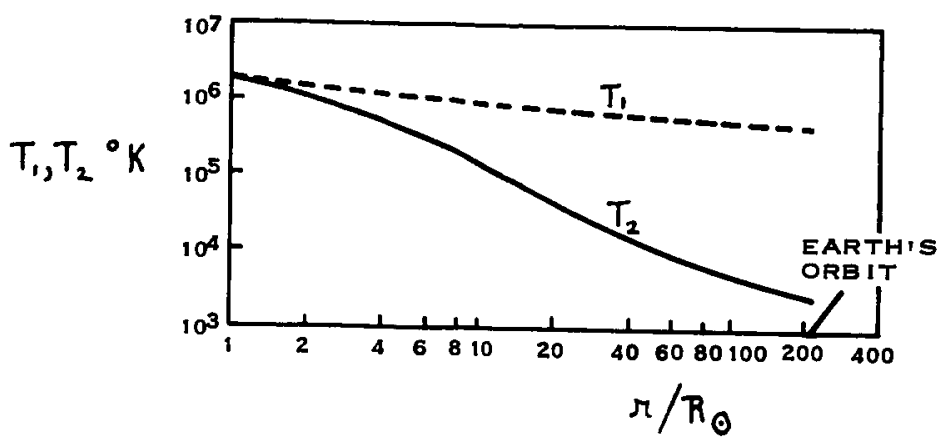

Figure VII. The electron temperature $T_{1}\left({ }^{\circ} \mathrm{K}\right.$, broken line $)$ and proton temperatu $\mathrm{reT}_{2}(\mathrm{O} \mathrm{K}$, solid line) as a function of radial distance (in units of $R_{\odot}=7 \times 10^{10} \mathrm{~cm}$ ) from the center of the sun. 


\section{SOLUTION OF THE EQUATION OF MOTION FOR THE HEAVY IONS}

\section{A. General Discussion}

The diffusion equations ( 23 ) and ( 24 ) are non-linear first order differential equations from which we desire to obtain solutions $V=V(\xi)$ over the range $1 \leqslant \xi \leqslant 5$. In principle, each one of these equations will have a single valued solution (for specified values of the quantities $F_{1}, F_{2}, \ldots . F_{5}$ ), provided a boundary value for $V \quad$ (or $d V / d \xi$ ) is given at some point, such as $\xi=1$. It should however again be emphasized that the basic flow properties of the ionized hydrogen background which appear in these equations (such as $U, r, T_{1}$, and $T_{2}$ ) are themselves not generally specified in closed form, but must be obtained numerically from point to point by solving transcendental equations. In general therefore, analytical solutions for $V=V(\xi)$ cannot be readily derived in closed form over the entire range of integration, and the diffusion equations will have to be integrated numerically on a computer. Before actually discussing these numerical solutions in other sections of this chapter, it is however possible and useful to answer the following question: if a typical heavy ion is injected near the base of the corona, will it either move upward, remain stationary in equilibrium, or fall back deeper toward the solar interior? More specifically, we will derive 
an approximate mathematical relation between the basic physical quantities involved $\left(M_{2}, M_{3},\left(e_{3} / e\right)^{2}, T_{0}, g\left(r_{0}\right)\right.$, etc...) giving the condition required for such an ion to start diffusing upward in the corona.

It has already been mentioned in Chapter III that, for small values of $\xi$, the transcendental equation ( 28 ) derived by Parker for $U$, in the case of an isothermal corona, has the following approximate asymptotic solution * in closed form:

$$
U=\frac{1}{\xi^{2}} e^{-\left(\frac{H}{\xi}+U_{2}^{2}\right)}
$$

where $\mathrm{H}$ and $U_{2}^{2}$ are constants which have been defined already. This expression for $U$ (along with the corresponding asymptotic expression for $r$, which follows directly from the equation of conservation of mass) will now be substituted into the first diffusion equation (23) to leave $V=V(\xi)$ as the only dependent variable; since the above asymptotic expression for $U$ is only accurate near $\xi \sim 1$,

\footnotetext{
* A corresponding asymptotic solution was not reported to exist by Sturrock and Hartle for their model; this discussion will therefore be limited to the model of Parker.

** For $T_{1}=T_{2}=T_{0}=1 \times 10^{6}{ }^{\circ} \mathrm{K}$, this approximate asymptotic solution is in error (compared to the exact solution obtained by solving the transcendental equation numerically) by less that $0.1 \%$ at $r=r_{0}, 6.0 \%$ at $r=2 r_{0}$, and $22.8 \%$ at $r=3 r_{0}$.
} 
it will be assumed that the ions have not yet reached thermal equillibrium with the background, and the second diffusion equation (24) will not be used. In any case, it will later be seen that both diffusion equations give very nearly the same solution for $V(\xi)$ at $\xi \sim 1$.

The resulting asymptotic diffusion equation will be further simplified by making the following additional assumptions, which are valid near the base of the corona $(\xi \sim 1)$ :

(1) The effect of the convective acceleration of the hydrogen background on the radial electric field is small compared to the effect of the other terms, since $U$ and $d U / d \xi$ are both small near $\xi=1$ : $F_{2} U \frac{d U}{d \xi} \ll \frac{F_{1}}{\xi^{2}}$ and $F_{2} U \frac{d U}{d \xi} \ll F_{3} r \log \frac{F_{4}}{\sqrt{r}}\left[\sum_{x=1}^{\infty} \frac{(-1)^{x+1}(2)^{x}(U-\gamma)^{2 x-1}}{(x-1) !(2 x+1)}\right]$

(2) If the ion is just barely able to diffuse upward in the corona, the kinetic friction force will have to be as great as possible to overcome the downward force of gravity; for given background conditions, this will occur if $V \ll U$.

(3) For the temperatures of interest in solar physics, $U \ll 1$

This will allow us to linearize the collisional term in the diffusion equation. We then obtain:

$$
V \frac{d V}{d \xi}=-F_{1}+\frac{2}{3}\left(F_{3} \log F_{4}\right) U
$$

At this point, the reader will be reminded that, with the model of Parker, $F_{1}, F_{3}$, and $F_{4}$ are constants given by the following 
formulas:

$$
\begin{aligned}
& F_{1}=r_{0} g\left(r_{0}\right)\left(\frac{M_{2}}{4 k T_{0}}\right)\left[1-\frac{1}{2}\left(\frac{l_{3}}{l}\right) \frac{M_{2}}{M_{3}}\right] \\
& F_{3}=\frac{2 r_{0} M_{0} e^{4}}{\left(k T_{0}\right)^{2}} \sqrt{\frac{\pi}{2}}\left(\frac{l_{3}}{l}\right)^{2}\left(\frac{M_{2}}{M_{3}}\right)^{2}\left(1+\frac{M_{3}}{M_{2}}\right) \\
& F_{4}=\frac{3}{2}\left(\frac{k T_{0}}{l^{2}}\right)^{3 / 2} \frac{1}{\sqrt{2 \pi M_{0}}}
\end{aligned}
$$

The necessary criterion for the ion to diffuse upward is that

$$
\begin{aligned}
& V \frac{d V}{d \xi}>0 . \text { Therefore, } \\
& \qquad \frac{2}{3}\left(F_{3} \log F_{4}\right) U\left(F_{1}\right.
\end{aligned}
$$

This may be made physically more meaningful by substituting the above formulas for $F_{1}, F_{3}, F_{4}$, and $U$. The resulting inequality is rather complicated and still not too illuminating at this stage, so that we will now restrict ourselves to an ion which is heavy enough (and not too strongly ionized) so that $M_{2} / M_{3} \ll 1$ and $\left(e_{3} / e\right) M_{2} / M_{3} \ll 1$.

The criterion for upward diffusion of the ion will now become, approximately,

$$
\frac{1}{M_{3}}\left(\frac{\ell_{3}}{l}\right)^{2}>\sqrt{\frac{2}{\pi}} \frac{3 k T_{0} g\left(\Omega_{0}\right)}{16 m_{0} l^{4} U \log \left[\frac{3}{2}\left(\frac{k T_{0}}{l^{2}}\right)^{1 / 2} \frac{1}{\sqrt{2 \pi M_{0}}}\right]}
$$

where the left hand side of the inequality depends on the physical characteristics of the ion only $\left(M_{3},\left(e_{3} / e\right)\right)$, while the right hand side depends on the physical characteristics of the background only $\left(n_{0}, T_{0}, U=U\left(T_{0}\right)\right)$. This allows us to state the following results:

(1) For given background conditions, a heavy ion may move away from the sun if a sufficient number of its atomic electrons have 
been ionized.

(2) A given ion may move away from the sun if the temperature $T_{0}$ or the number density $m_{0}$ of the corona at $\pi=\pi_{0}$ are high enough.

The following numerical example will serve to clarify the meaning of the above statements:

Let us consider an ionized atom of iron injected into the base of the corona maintained at a temperature $T_{0}=1 \times 10^{6} \mathrm{~K}$, and let us find the minimum level of ionization required for upward convection of the ion. After substituting the appropriate values of $m_{0}, g\left(r_{0}\right), M_{3}$ (neglecting the mass of the electrons and the atomic binding energy), we find

$$
\left(\frac{e_{3}}{l}\right)^{2}>74.5
$$

or, approximately, $\left(\frac{\ell_{3}}{l}\right)>8.63$; however, $\left(\frac{\ell_{3}}{\ell}\right)$ must be an integer, so that we must take $\left(\frac{l_{3}}{l}\right) \geqslant 9$. This result agrees exactly with spectroscopic observations of the coronal lines: the least ionized iron atom observed in the corona was found to be FeX, with 9 electrons stripped off. The following $\mathrm{Fe}$ ions have also been observed in the corona: Fe XI, Fe XII, Fe XIII, Fe XIV, and FeXV.

It should be remembered that these results only apply near the base of the corona; the next step in the solution is to actually obtain the velocity of the ions in space for $r>\pi_{0}$, and this will be discussed in sections $C$ and $D$ of this chapter. 


\section{B. Selection of Heavy Ions of Astrophysical Interest}

Summarizing what has been accomplished so far, we have first derived general diffusion equations from which the dynamical behavior of heavy charged particles found in the solar corona may be predicted. We have also derived an approximate relation from which we may compute the minimum charge such particles must carry to be convected against gravity. We now want to actually solve the diffusion equations to obtain $V=V(\xi)$ for a number of charged particles of special interest to solar physics. It has already been mentioned in Chapter I (Definition of the Problem) that spectroscopic observations have shown that the solar atmosphere is extremely rich in such charged particles; the purpose of this section is therefore to choose a representative sample of charged particles to be used in numerical calculations.

Although some chemical compounds(mostly diatomic colecules like $\mathrm{CN}, \mathrm{C}_{2}, \mathrm{CH}, \mathrm{NH}$, etc. ) have been discovered to exist in the lower regions of the solar atmosphere, the majority of these molecules cannot exist in the solar corona because they would immediately be decomposed by violent collisions resulting from the high temperatures of the gas (Flammarion, 1964). We will therefore ignore the presence of stable charged molecular fragments, and limit 
our attention to ionized atoms.

We must therefore answer the following two questions: first, which elements should be selected and, second, whichions of these elements should be considered.

It is generally considered (Kuiper, 1958) that the sun consists, by mass, of $82 \%$ Hydrogen, $17 \%$ Helium, and $1 \%$ for all other ele ments combined together.. We will therefore select Helium as the first element of our sample, because of its overwhelming abundance. The next logical choice would seem to be Carbon * since it is, after Helium, the most abundant element in the sun. However, it is still a comparatively "light" element, and it will therefore be more interesting to focus our attention on elements with atomic numbers larger than 15 or so. If the estimated solar abundances of these "heavy" elements are compared (Kuiper, 1964), it will quickly become obvious that Iron is the most important species. (For instance, the abundance of Iron is approximately 6.3 times larger than that of its nearest competitor, Sulphur, and approximately 372 times larger than that of the next heavier element, Cobalt). We therefore select

* The intervening light elements (lithium, beryllium, and boron) are known to be abnormally rare in the solar atmosphere, probably because of the ease with which they may be destroyed in the solar interior by nuclear reactions; they are therefore of no special interest to this study. 
Iron as the second element in our sample.

The selection of the ions of Helium and Iron is obtained in two steps: first, atomic physics handbooks (such as those prepared by Moore, 1952) are consulted to see how many ionization stages are possible for these two elements: this gives $\mathrm{He}$ II and ${ }_{2} \mathrm{He}^{4}$ for Helium, and Fe II, Fe III, ...Fe XVIII for Iron. Second, we eliminate from this group ions which either do not satisfy the "diffusion condition"derivedin Section A of this Chapter, or which have not been observed spectroscopically in the corona. This leaves the following ions:

$$
T_{0}=1 \times 10^{60} \mathrm{~K} *:{ }_{2} \mathrm{He}^{4}
$$

Fe X, Fe XI, Fe XII, Fe XIII, Fe XIV, Fe XV

$$
T_{0}=2 \times 10^{6} 0_{\mathrm{K}}: \mathrm{He} \mathrm{II},{ }_{2} \mathrm{He}^{4}
$$

Fe X, Fe XI, Fe XII, Fe XIII, Fe XIV, Fe XV

Finally. in the case of Iron. calculations will only be presented for $\mathrm{Fe} \mathrm{X}$ and $\mathrm{Fe} \mathrm{XV} \mathrm{(the} \mathrm{two} \mathrm{extreme} \mathrm{cases)} \mathrm{since} \mathrm{the} \mathrm{other} \mathrm{in-}$ tervening ions may be expected to give results falling in between those of Fe X and Fe XV.

* This selection of $T_{0}$ will be explained in the next section. 


\section{Model of Parker}

The next step in solving the diffusion equations, for each ion mentioned in the previous section, is to evaluate numerically the coefficients $F_{1}, F_{2}, \ldots F_{5}$. With the isothermal model of Parker discussed in this section $\left(T_{1}=T_{2}=T_{0}=\right.$ constant $), F_{1}, F_{2}, \ldots . F_{5}$ are all constants and may be computed from the following parameters: $\pi_{0}, g\left(r_{0}\right), T_{0}, M_{2},\left(\frac{e_{3}}{e}\right), M_{3}, M_{0}$, and $e$; of these, $e$, r. $g\left(h_{2}, M_{2}\right.$, and $M_{0}$ are basic physical constants common to all cases, and $\left(\frac{\ell_{3}}{l}\right), M_{3}$ depend on the particular ion under study; however, $T_{0}$ remains undetermined. It has already been mentioned in Chapter III that, since the isothermal model is only an approximation to the real temperature distribution, no single value of $T_{0}$ is entirely satisfactory for all cases; estimates of $T_{0}$ based on experimental observations of the corona apparently depend on what physical quantitities are actually being measured. A good review of this perplexing problem was given by Allen (1963). For instance, early temperature measurements based on line widths were about $2.0 \times 10^{6} \mathrm{O}$ (Allen, 1954); more recent estimates based on the same method seem to be about $2.4 \times 10^{6} \circ \mathrm{K}$ (Billings, 1959) or more (Jarrett and von Klüber, 1961). On the other hand, temperatures derived from the degree of ionization, and therefore dependent on calculated ionization and recombination coefficients, have 
consistently turned out to be lower, of the order of $0.8 \times 10^{6} \circ \mathrm{K}$. It will later be seen in this section that such differences in $T_{0}$ have a marked influence on the results.

Other experimental determinations of $T_{0}$ have persistently given two sets of values, a "low" and a "high". The results may be summarized as follows:

Line Widths (Unsöld,1960) Kinetic Temperature. $T_{0}=$ $2.4 \times 10^{6} \circ \mathrm{K}$

Scale Height (Unsöld, 1960; Kinetic Temperature. $T_{0}=$ Pottasch, 1960) 1. $6 \times 10^{60} \mathrm{~K}$

Ionization (Allen, 1954)

Electron Temperature. $T_{0}=$ $0.8 \times 10^{60} \mathrm{~K}$

Radio Brightness in $\mathrm{m}$ Band Electron Temperature. = (Newkirk, 1959) $\quad 0.8 \times 10^{6} 0^{\circ} \mathrm{K}$

It will be noticed that the heavy particles tend to give high temperatures and the electrons low temperatures, hence some consideration was given (Liège Colloquium, 1961) to the suggestion that this difference was real. It was proposed that the mechanical energy that produced coronal heating was applied to the heavy particles, while the energy loss was mostly associated with the electrons. Unfortunately, this is in contradiction with the results of Sturrock and Hartle (See Chapter III), who have shown that, on theoretical grounds, the temperature of the electrons should be higher, not lower, than the temperature of the protons. 
Instead of attempting to resolve this difficulty at this time, we will simply present two sets of results corresponding respectively to $T_{0}=I \times 10^{6} \mathrm{o} \mathrm{K}$ and $T_{0}=2 \times 10^{6 \circ \mathrm{K}}$.

The constants $F_{1}, F_{2}, \ldots F_{5}$ may now be evaluated and are given in Table II for the case $T_{1}=T_{2}=T_{3}=T_{0}$; in the case $T_{3}=0, T_{1}=T_{2}=T_{0}$, the constants $F_{1}, F_{2}, \ldots . F_{4}$ have the same value as for the case $T_{3}=T_{0}$, while $F_{5}=0$.

Computer programs were then prepared, in order to integrate the diffusion equations (23) and (24) numerically on a CDC 160-A computer, so as to obtain $V=V(\xi)$, as well as $U$ and $r$ from $\xi=I$ to $f=5$. As it was explained already in Chapter III, the transcendental equation (28), which must be solved for $U$ from point to point by an iteration subroutine, has a singular point at $\xi=\xi_{c}=\frac{H}{2} *$. The integration was therefore carried out in two steps, from $\xi=I$ to $\xi=\frac{H}{2}-\varepsilon$, and from $\xi=\frac{H}{2}+\varepsilon$ to $\zeta=5$, where $\varepsilon$ is a small number. A typical program listing is reproduced in Figure VIII for the case of ${ }_{2} \mathrm{He}^{4}$ ions at $T_{3}=0$ and $T_{0}=I \times 10^{6} \circ \mathrm{K}$. The other cases (for

* At the singular point, where the upper and lower branches of equation (28) come together, there are, mathematically, two possible solutions for $\frac{d u}{d \xi}$, one negative, one positive, for one value of $U=U_{c}$; however, only the positive solution for $\frac{d U}{d f}$ is
physically acceptable. 


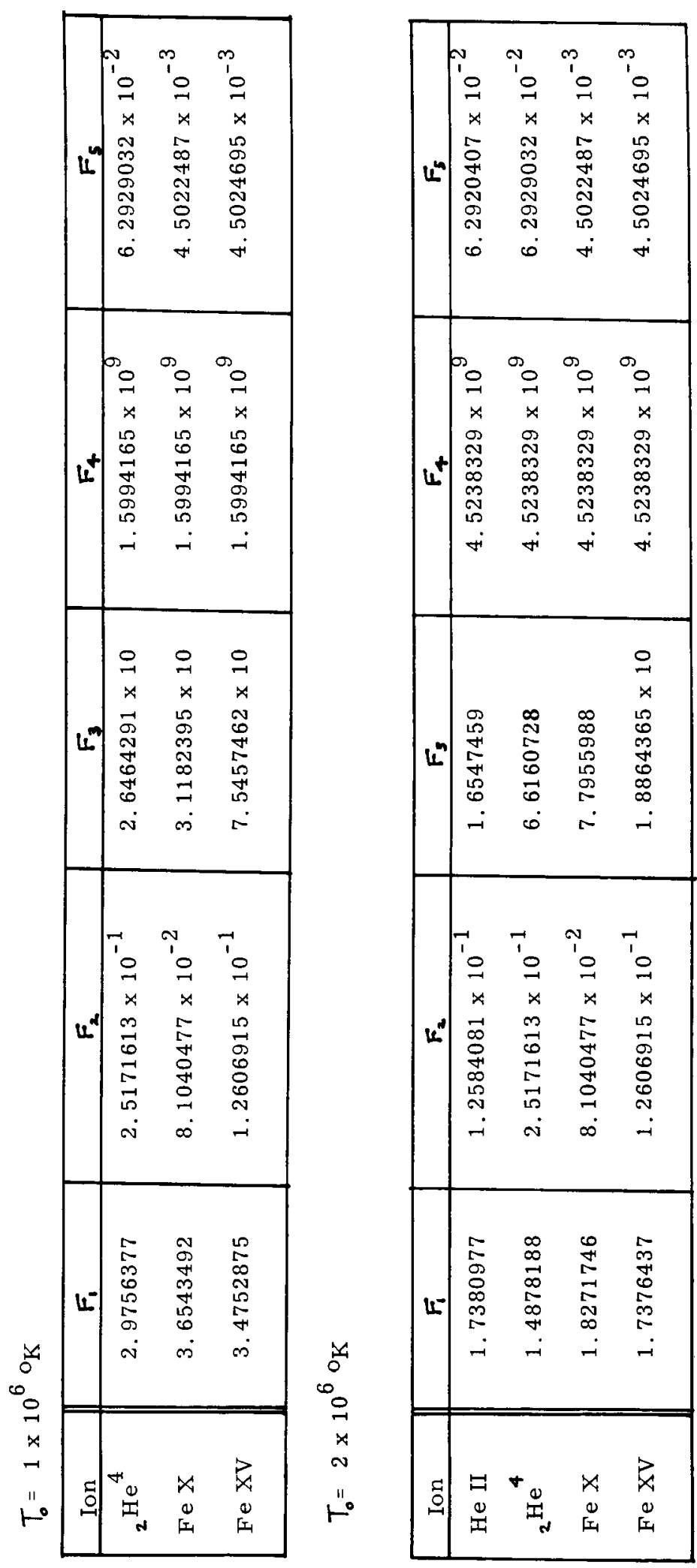

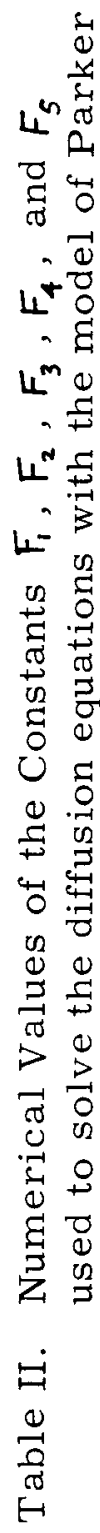



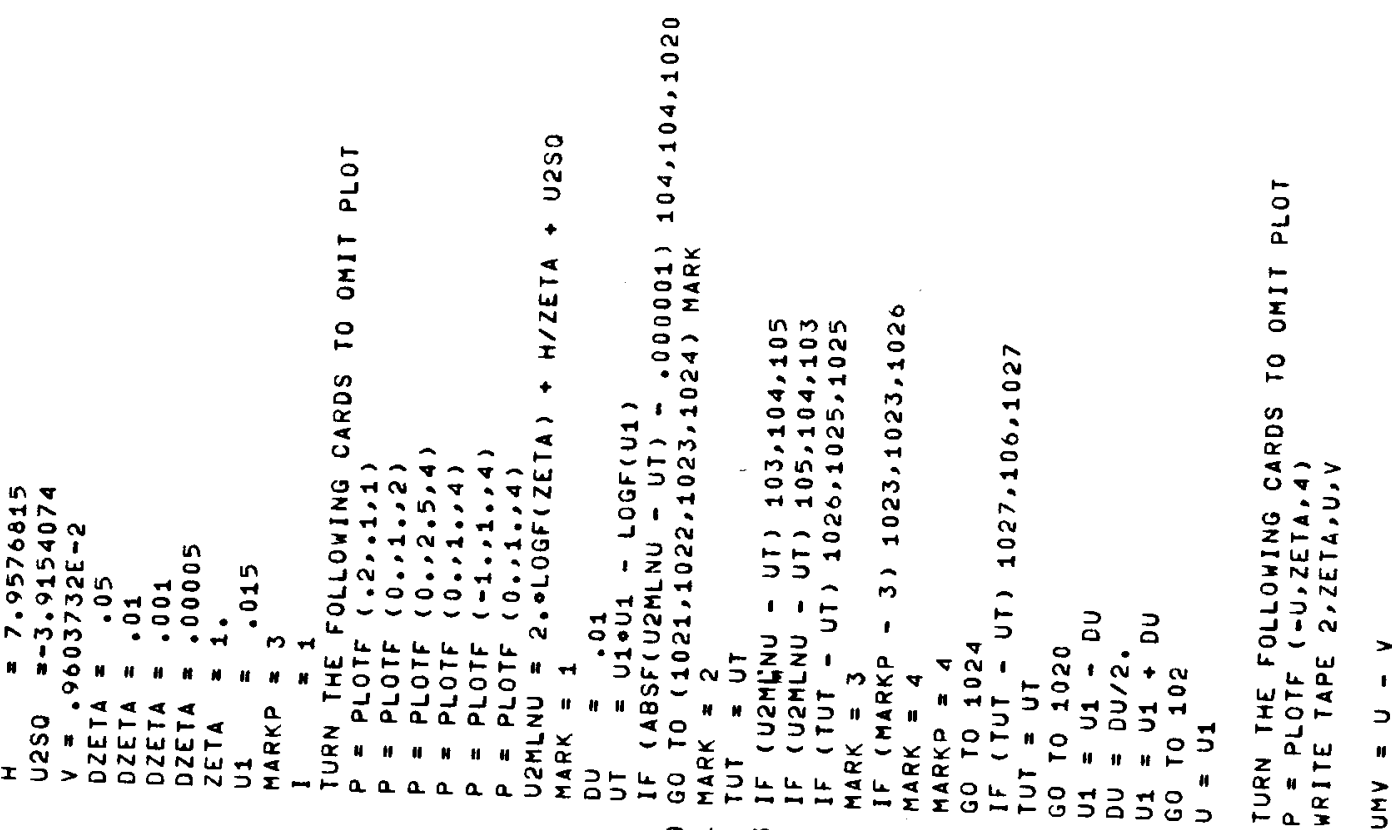

- 000

o

함

요웜

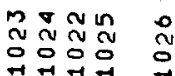

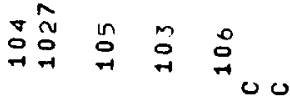

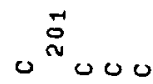


$0 \stackrel{\text { N }}{0}$

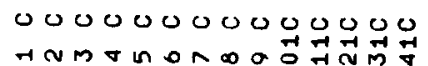
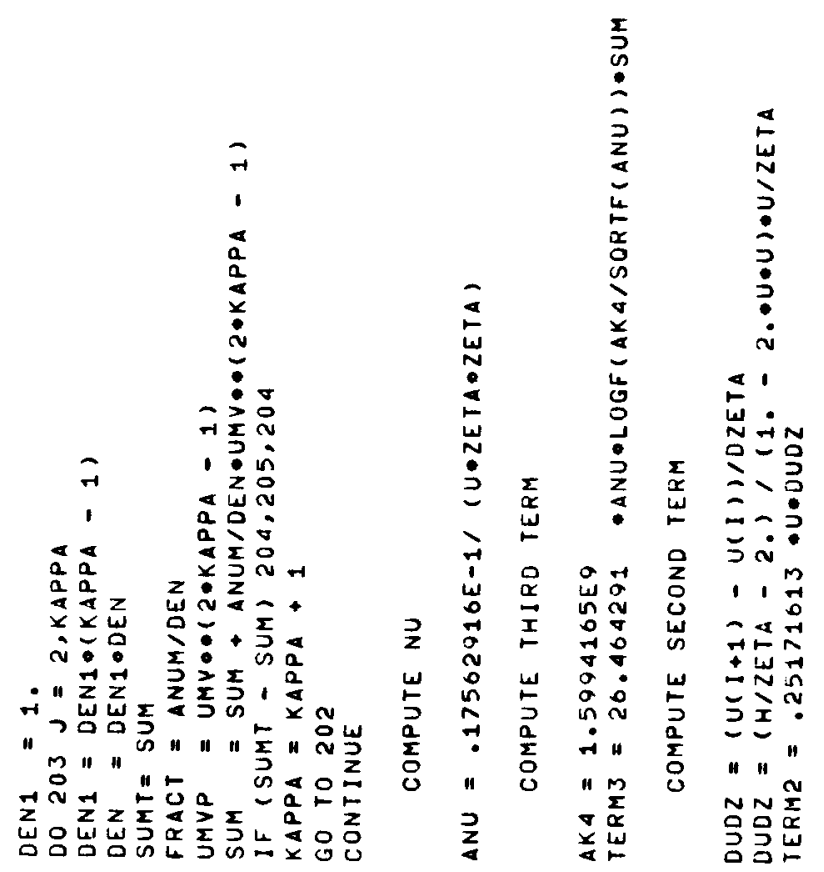

i

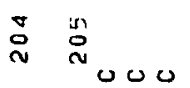

000

0000

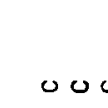

Figure VIII

Computer Program Listing for the case of ${ }_{2} \mathrm{He}^{4}$ ions at $T_{3}=0$ and $T_{0}^{2}=1 \times 10^{6} \mathrm{~K}$, One-Fluid Model of Parker 
$T_{3}=0$ ) may be obtained by substituting the appropriate numerical values for the various input quantities: $H$ and $U_{2}^{2}$ (functions of $T_{0}$ only), $F_{1}, \ldots F_{4}$ (functions of $\ell_{2}, M_{3}$, as well as $T_{2}$ ), and $V_{0}$, the boundary value of $V$ at $\xi=1$. In the case $T_{3} \neq 0$, an additional term (TERM4 in computer language) was added to take into account the effect of pressure gradient due to the finite temperature of the ions.

The boundary value $V_{0}$ referred to above was obtained by means of a relaxation method which will now be described.

Although we speak, in each physical case of interest, of one boundary value for $V$, we know that this is only a oversimplification of what really takes place at the base of the corona: because of the turbulent conditions and convection currents which are thought to exist in the chromosphere, the ions will be injected into the corona with a certain distribution or spread in velocity, rather than with a well-defined radial velocity component: as they start interacting with the coronal electrons and protons, those with "low" velocities will be accelerated upward faster than those with "high" velocities; however, since the two dynamical models of the corona used in this thesis assume that the electrons and protons have the same singlevalued flow velocity at any point, the ions will, after a certain characteristic relaxation distance $\lambda_{\Omega}$, acquire the same common 
flow velocity $V$.A large number of numerical calculations have shown that $\frac{\lambda_{\lambda}}{r_{0}} \ll I$ in all cases of interest. The computer may therefore be used as a tool to simulate, mathematically, the physical relaxation process which takes place as the ions attain a common flow velocity. The proper boundary value to be used for $V$ at $\xi=$ I may then be obtained by solving the diffusion equations numerically from $\xi=I-\lambda_{r}$ to $\xi=I$, using a number of reasonable initial values for $V$ at $\xi=I-\lambda_{\Omega}$, and letting the resulting solutions relax to a common value.

Graphs of $V=V(\xi)$ and $U=U(\xi)$ are shown in Figure IX $\left(T_{1}=T_{2}\right.$ $\left.=\mathrm{I} \times 10^{6} \circ \mathrm{K}\right)$ and Figure X $\left(T_{1}=T_{2}=2 \times 10^{6 \circ} \mathrm{K}\right)$ for each ion of interest, and for the cases $T_{3}=0$ (broken lines) and $T_{3}=T_{2}$ (solid lines).

The above flow velocities were then used to compute, with the equations of conservation of mass, the relative abundance $\frac{\left(m_{3} / m\right)_{\xi}}{\left(m_{3} / m\right)_{\xi=1}}$ for each type of ion; these results are given in Figures XI and XII. 


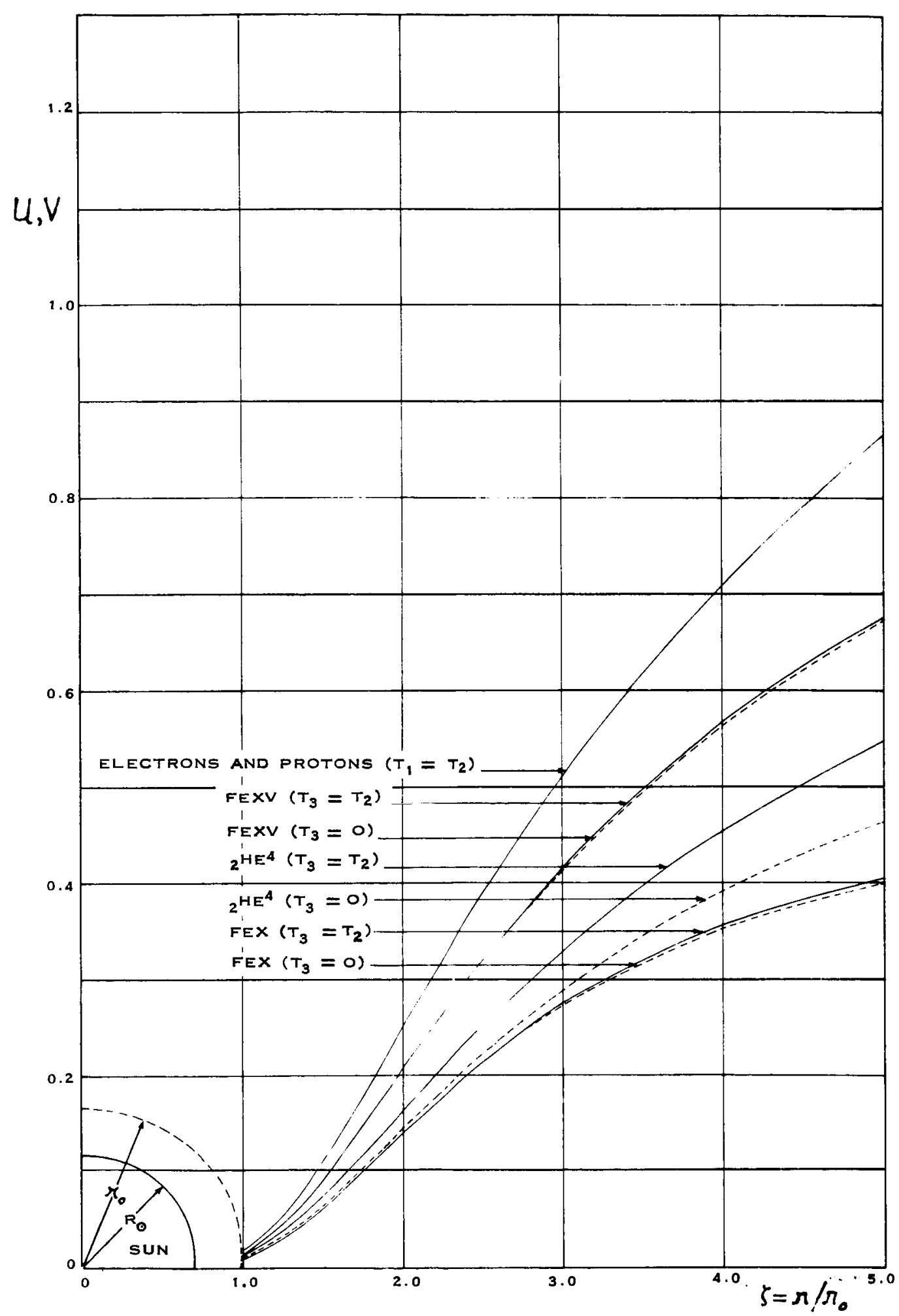

Figure IX. Graph of the Flow Velocity of the Electrons, Protons and Ions as a Function of the Distance from the Sun. One-fluid Model of Parker, $T_{1}=T_{2}=T_{0}=1 \times 10^{6 \circ} \mathrm{K}$. 


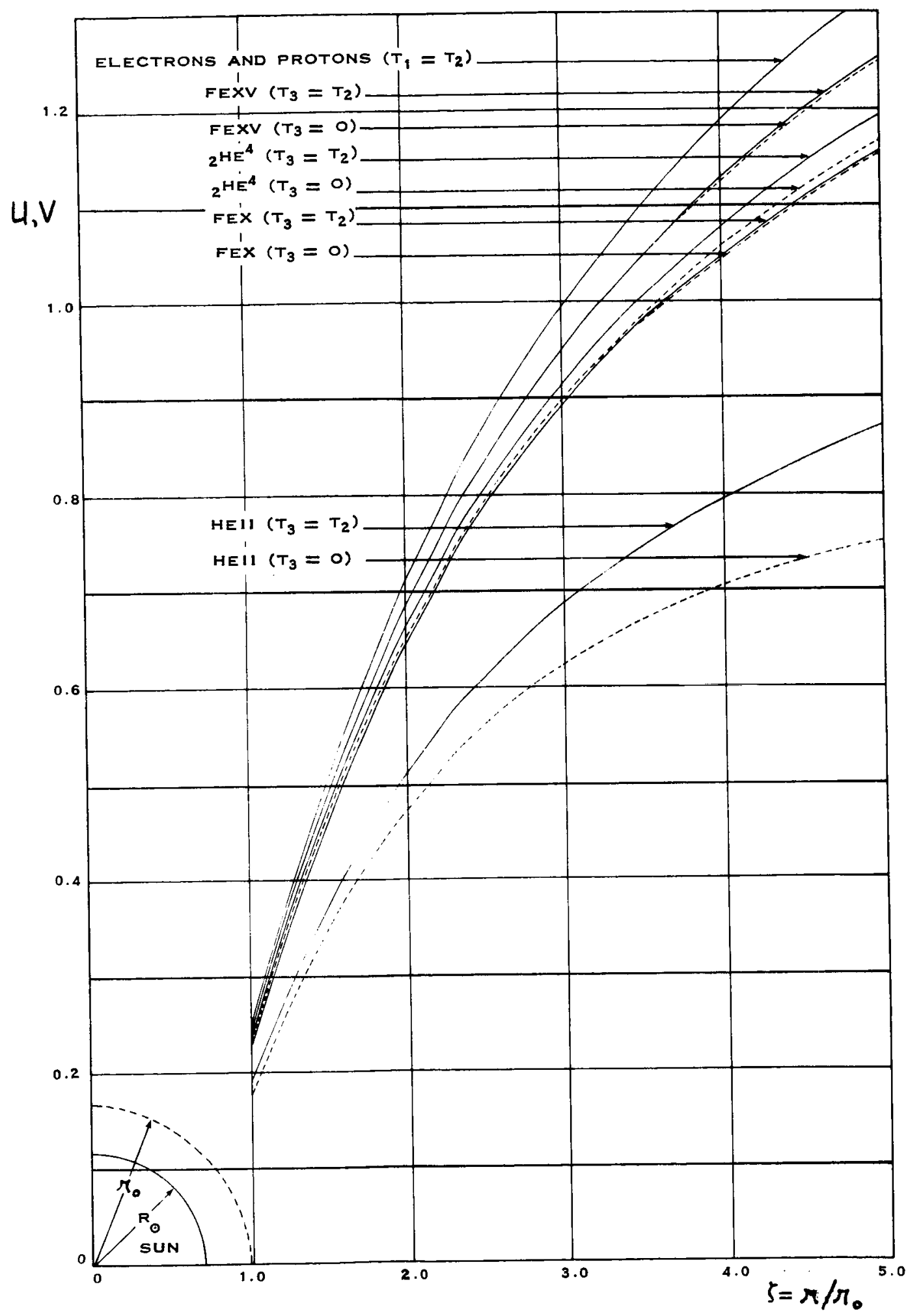

Figure X. Graph of the Flow Velocity of the Electrons, Protons and Ions as a Function of the Distance from the Sun. One-Fluid Model of Parker, $T_{1}=T_{2}=T_{0}=2 \times 10^{6} \circ \mathrm{K}$ 


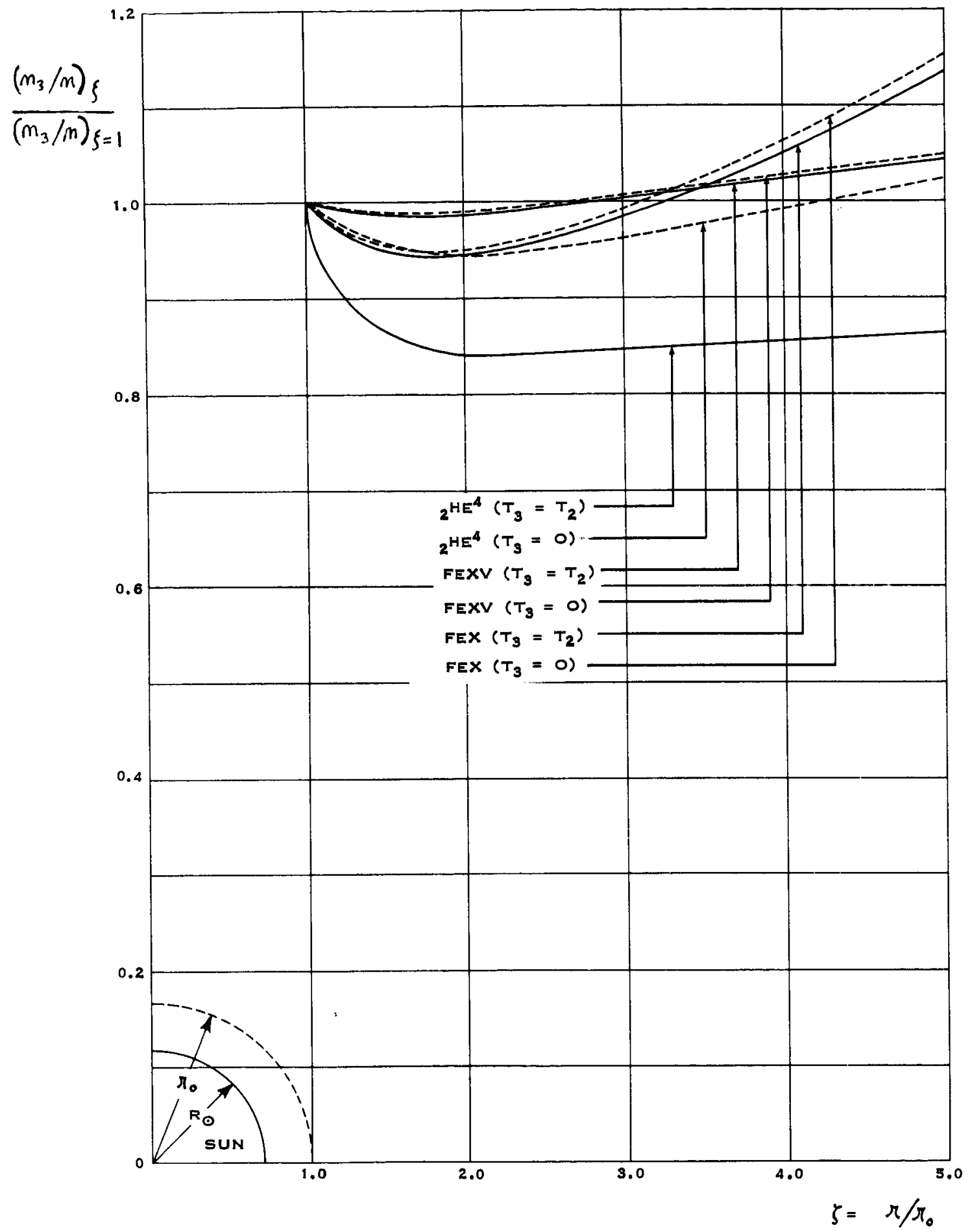

Figure XI. Graph of the Relative Abundance of the Ions as a Function of the Distance from the Sun. OneFluid Model of Parker $T_{1}=T_{2}=T_{0}=1 \times 10^{6}{ }^{\circ} \mathrm{K}$. 


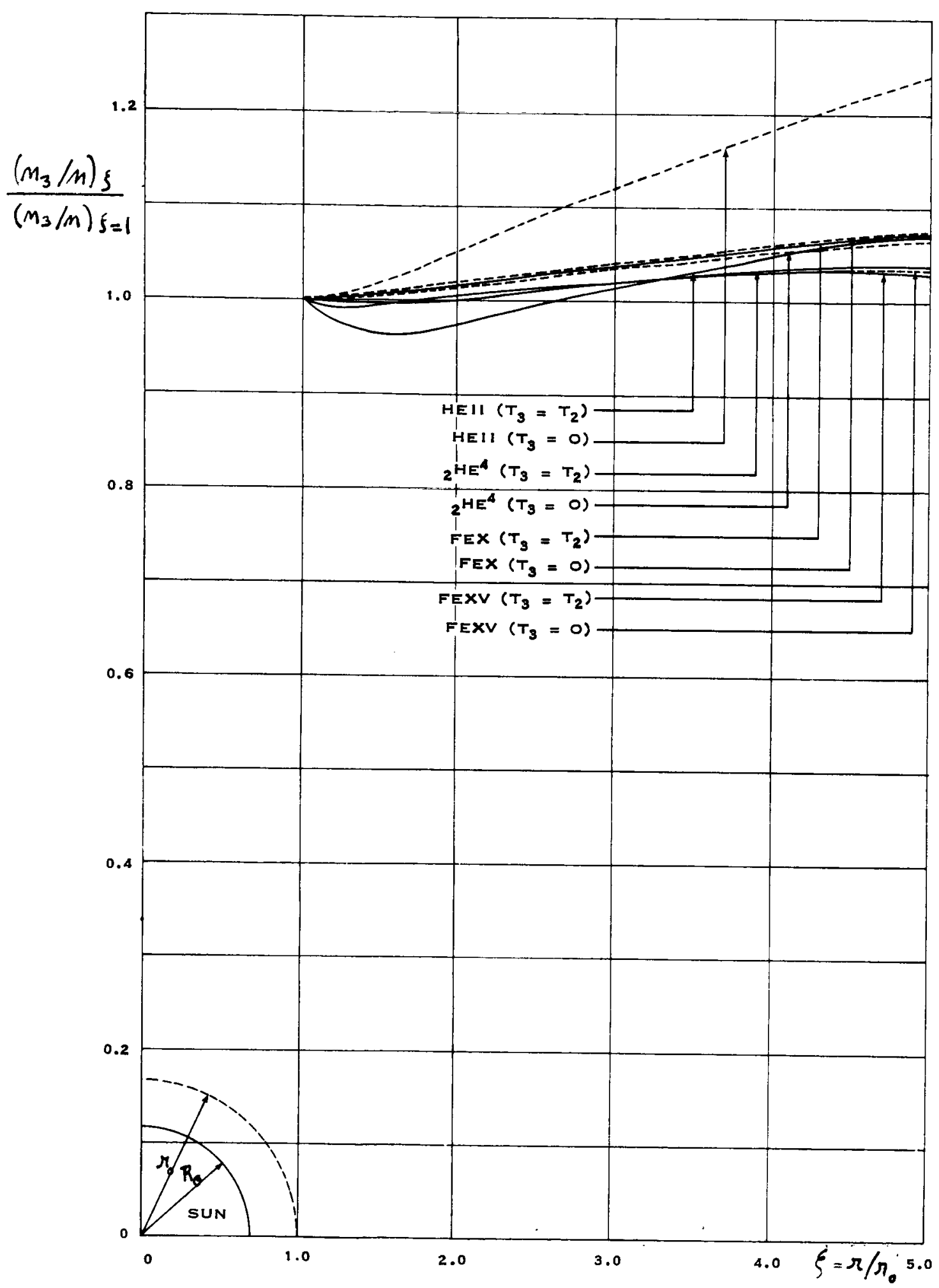

Figure XII. Graph of the Relative Abundance of the Ions as a Function of the Distance from the Sun. One-Fluid Model of Parker $T_{1}=T_{2}=T_{0}=2 \times 10^{6} \mathrm{O}_{\mathrm{K}}$. 
D. Model of Sturrock and Hartle

Although the diffusion equations (23), (24) have the same general form regardless of which theoretical model is used to describe the coronal background, they are in reality far more difficult to solve rigorously if the model of Sturrock and Hartle is used instead of the isothermal model of Parker, since:

(1) The temperatures $T_{1}$ and $T_{2}$ are no longer equal and constant throughout the corona.

(2) The polytrope indices $\alpha_{1}$ and $\alpha_{2}$ are no longer equal and constant throughout the corona.

(3) The dimensionless background flow velocity $U$ must be obtained numerically from point to point by solving a transcendental equation which is far more complex than the one used previously.

An approximate scheme intended to use, as much as possible, the techniques derived previously for the model of Parker, was therefore devised and will now be described. The basic approach consists in dividing the region of the corona under study in a finite number of regions (not necessarily of equal width) within each of which $T_{1}, T_{2}$, $\alpha_{1}$ and $\alpha_{2}$ may be assumed to be constant. The accuracy of this 
technique should, in principle, improve as the number of such distinct coronal regions is increased. However, it may easily be seen that this will quickly lead to very laborious matching problems when passing from one region to the next. In practice, therefore, a compromise was reached, which was based on the fact that the variable temperatures $T_{1}$ and $T_{2}$ appear as dimensionless ratios $\left(T_{2} / T_{1}\right.$ and $T_{1} / T_{0}$ ) in the definitions of $F_{1} \ldots F_{5}$; since $T_{2}$ does not vary as rapidly with $\pi$ as $T_{1}$, the distinct coronal regions were selected by considering $T_{2} / T_{1}$ as the basic criterion; this led to the selection of the following 5 regions: $1 \leqslant r / R_{\odot} \leqslant 2,2 \leqslant r / R_{\odot} \leqslant 4,4 \leqslant \pi / R_{\odot} \leqslant 5$, $5 \leqslant r / R_{\odot} \leqslant 6$, and $6 \leqslant r / R_{\odot} \leqslant 7$, where $T_{1} / T_{2}$ varies from 1.0 to 3.3 approximately.

The rest of the treatment is identical to the approach taken with the model of Parker, already described in Section $\mathrm{C}$ of this chapter.

A graph of $V=V(\xi)$ and $U=U(\xi)$ is shown in Figure XIII; a graph of the relative ionic abundance is given in Figure XIV. 


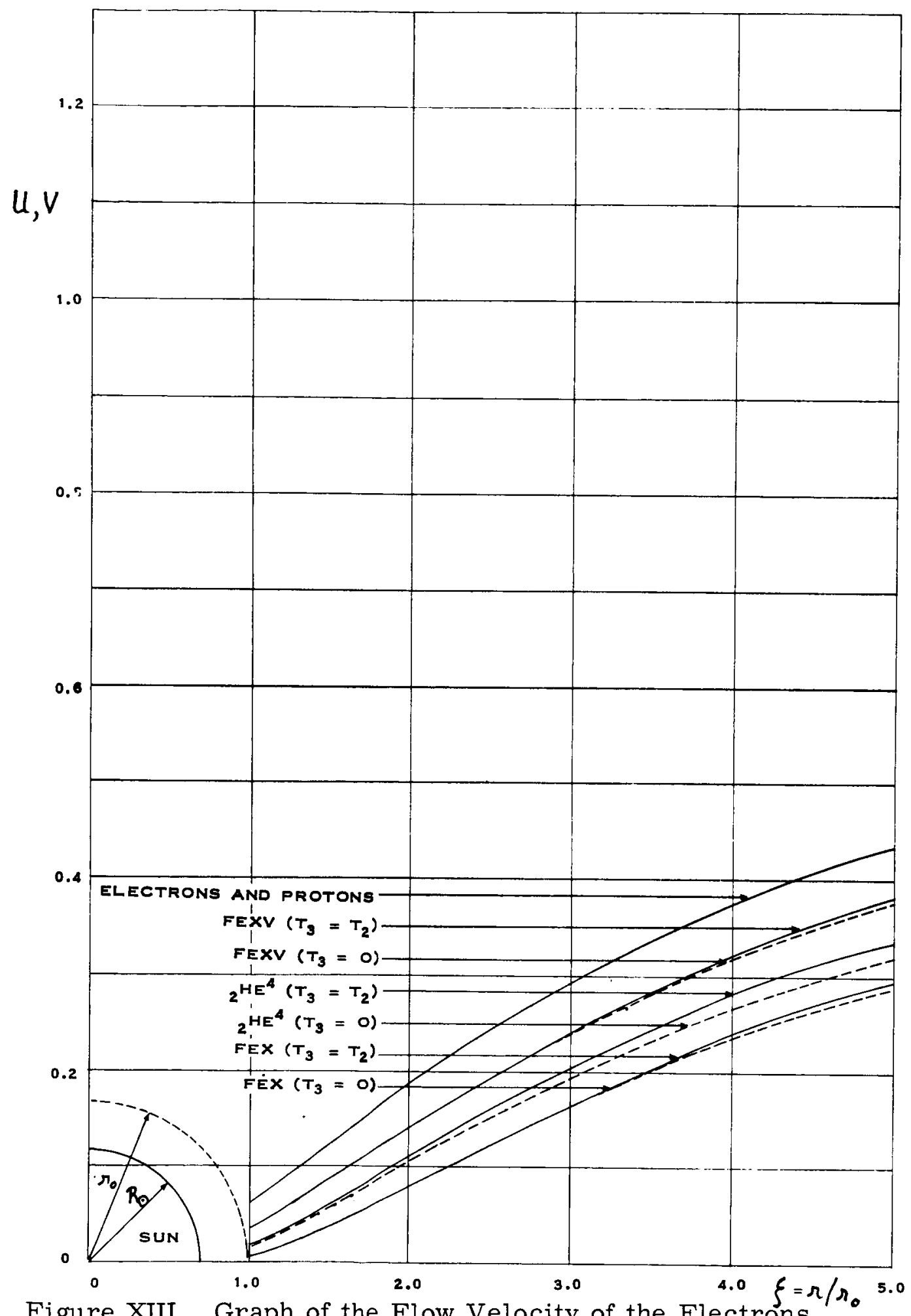

Figure XIII. Graph of the Flow Velocity of the Electrons, Protons and Ions as a Function of the Distance from the Sun. Two-Fluid Model of Sturrock and Hartle, $T_{0}=2 \times 10^{6} \mathrm{o}_{\mathrm{K}}$. 


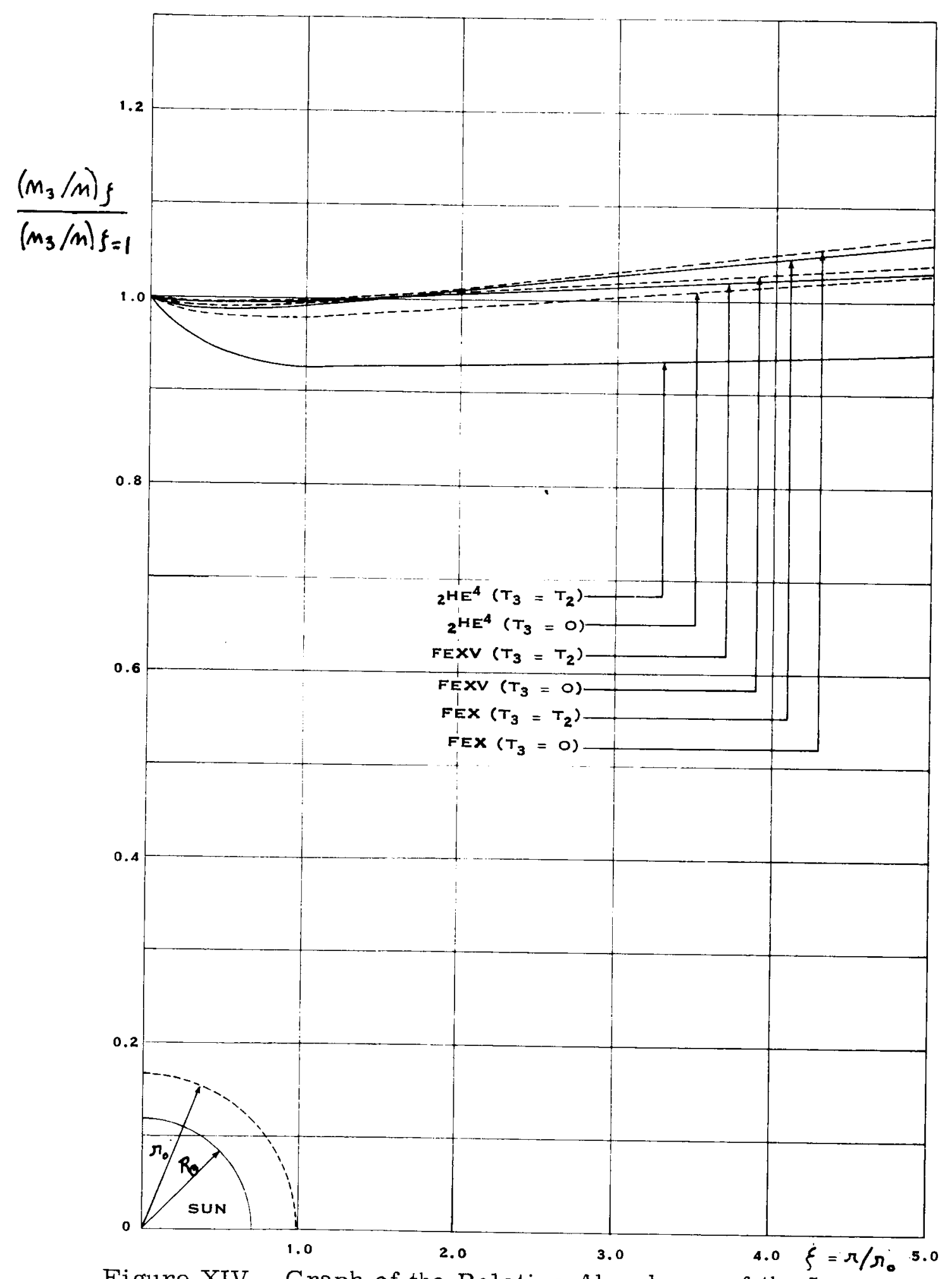

Figure XIV. Graph of the Relative Abundance of the Ions as a Function of the Distance from the Sun. Two-Fluid Model of Sturrock and Hartle, $T_{0}=2 \times 10^{6}{ }^{0} \mathrm{~K}$. 


\section{CONCLUSIONS}

It has been shown that it is theoretically possible for heavy ionized atoms to diffuse upward in the solar corona, provided certain conditions are met between the charge and mass of these particles and the temperature of the ionized hydrogen background. A representative sample of ions satisfying these conditions was selected,and the diffusion equations were solved to obtain the flow velocity and relative abundance of these ions in the region of the corona extending as far as the lower solar wind, for two different theoretical dynamical models of the coronal background.

These results, which are presented graphically, have shown that

(1) In all cases, the ions will diffuse upward more slowly than the background. For given background conditions, the flow velocity of the ions will increase with the ionizing level of the atoms (Coulomb scattering cross-section), and decrease with the mass of the ions.

(2) For a given ion, the diffusion flow velocity will increase as the ionic temperature increases to attain thermal equilibrium with 
the background protons. This is especially noticeable in the case of light, slightly ionized atoms, such as He II; on the other hand, this effect is not very important in the case of massive, strongly ionized atoms, such as Fe XV.

(3) In general, the relative abundance of the ions in the corona will tend to increase slowly and gradually with height, after suffering a slight decrease near the bottom of the corona. At a given altitude, the abundance of a given ion will increase with the temperature (and flow velocity) of the background.

(4) In general, the ions will "follow" the motion of the background fluid more closely over the entire range of integration, if the coronal model of Sturrock and Hartle is used instead of the model of Parker. This will have the effect of reducing variations in the relative abundance of the ions. 


\section{APPENDIX I}

Exact calculation of $g(r)$

From elementary potential theory, the net acceleration of gravity $g(r)$ acting on a test particle of mass $M_{3}$ located in the solar atmosphere at a distance $r=R$ from the center of the sun will be

$$
g(R)=g\left(r_{0}\right)\left(\frac{r_{0}}{R}\right)^{2}+\frac{4 \pi G}{R^{2}} \int_{r_{0}}^{R} \rho r^{2} d r
$$

where the first term represents the contribution of the central solar core (of radius $\pi_{0}$ ), and the second term represents the contribution of the other particle in the solar atmosphere. We will now show that this second term is negligible compared to the first one, using, as an example, the asymptotic value obtained by Parker for $\rho$ (see Chapter III):

$$
\rho \simeq\left(M_{1}+M_{2}\right) m \simeq\left(M_{1}+M_{2}\right) M_{0} e^{H\left(\frac{r_{0}}{r}-1\right)}
$$

The resulting integral is rather difficult to evaluate exactly, so that we evaluate instead an upper bound to its value by taking

$$
\rho=\rho_{\text {mex }} \simeq\left(M_{1}+M_{2}\right) M_{0}
$$

This gives:

$g(R) \leqslant g\left(r_{0}\right)\left(\frac{r_{0}}{R}\right)^{2}+\frac{4 \pi G\left(M_{1}+M_{2}\right) M_{0}\left(R^{3}-r_{0}^{3}\right)}{3 R^{2}}$ 
At the Earth's Orbit, $R \sim 150 \pi_{0}$, and the second term amounts to about $3.61 \times 10^{-10}$ times the first term; closer to the sun, in the corona, the correction due to the second term is even smaller and may be neglected. 
APPENDIX II

Evaluation of $\left(\frac{\delta f_{3}}{\delta t}\right)_{C_{011}}$

We start from the expressions given on page 38 and 39 , substitut Maxwellian functions for $f_{1}$ and $f_{2}$ and evaluate the integrals:

$$
\begin{aligned}
\left(\frac{\delta f_{3}}{\delta t}\right)_{C_{0} 11}= & M_{0} \Gamma_{3}\left[-\frac{\partial}{\partial \vec{v}} \cdot\left\{f_{3} \frac{\partial}{\partial \vec{v}}\left[\left(\frac{M_{1}+M_{3}}{M_{1}}\right) \int \frac{d^{3} \vec{v}_{1} f_{1}\left(\vec{v}_{1}\right)}{\left|\vec{v}-\vec{v}_{1}\right|}+\left(\frac{M_{2}+M_{3}}{M_{2}}\right) \int \frac{d^{3} \overrightarrow{v_{2}} f_{2}\left(\vec{v}_{2}\right)}{\left|\vec{v}-\vec{v}_{2}\right|}\right]\right\}\right. \\
& \left.+\frac{1}{2} \frac{\partial^{2}}{\partial \vec{v} \partial \vec{v}}:\left\{f_{3} \frac{\partial^{2}}{\partial \vec{v} d \vec{v}}\left[\int d^{3} \overrightarrow{v_{1}}\left|\vec{v}-\vec{v}_{1} f_{1}\left(\vec{v}_{1}\right)+\int d^{3} \overrightarrow{v_{2}}\right| \vec{v}-\vec{v}_{2} \mid f_{2}\left(\overrightarrow{v_{2}}\right)\right]\right\}\right]
\end{aligned}
$$

This requires the evaluation of the following integrals:

1)

$$
\begin{aligned}
I_{1} & =\int_{-\infty}^{\infty} d^{3} \vec{v}_{1} \frac{e^{-a\left(\vec{v}_{1}-\vec{c}_{0}\right)^{2}}}{\left|\vec{v}-\vec{v}_{1}\right|} \\
& =\int_{-\infty}^{\infty} d^{3} \vec{V} \frac{e^{-a\left[v^{2}-2 \vec{v} \cdot \vec{c}_{0}+c_{0}^{2}+V^{2}+2\left(\vec{v}-\vec{c}_{0}\right) \cdot \vec{V}\right]}}{V} \\
& =e^{-a\left(\vec{v}-\vec{c}_{0}\right)^{2}} \int_{0}^{\infty} V^{2} d V \int_{0}^{\pi} \sin \theta d \theta \int_{0}^{2 \pi} \frac{d \varphi e^{-a\left[V^{2}+2\left(\vec{v}-\vec{c}_{0}\right) \cdot \vec{V}\right]}}{V} \\
& =2 \pi e^{-a\left(\vec{v}-\vec{c}_{0}\right)^{2}} \int_{0}^{\infty} V d V \int_{0}^{\pi} \sin \theta d \theta e^{-a\left[V^{2}+2\left|\vec{v}-\vec{c}_{0}\right| V \cos \theta\right]} \\
& =\frac{\pi e^{-a\left(\vec{v}-\vec{c}_{0}\right)^{2}}}{a\left|\vec{v}-\vec{c}_{0}\right|} \int_{0}^{\infty} e^{-a V^{2}}\left[e^{2 a\left|\vec{v}-\vec{c}_{0}\right| V}-e^{-2 a\left|\vec{v}-\vec{c}_{0}\right| V}\right] d V \\
& =\frac{\pi}{a\left|\vec{v}-\vec{c}_{0}\right|} \int_{0}^{\infty} e^{-a\left[\left|\vec{v}-\vec{c}_{0}\right|-V\right]^{2}} d V-\frac{\pi}{a\left|\vec{v}-\vec{c}_{0}\right|} \int_{0}^{\infty-a\left[\left|\vec{v}-\vec{c}_{0}\right|+V\right]^{2}} d V
\end{aligned}
$$

91 


$$
\begin{aligned}
& =\frac{\pi}{a\left|\vec{N}-\vec{c}_{0}\right|} \int_{-\left|\vec{N}-\vec{c}_{0}\right|}^{\infty} e^{-a x^{2}} d x-\frac{\pi}{a\left|\vec{v}-\vec{c}_{0}\right|} \int_{\left|\vec{v}-\vec{c}_{0}\right|}^{\infty} e^{-a x^{2}} d x \\
& =\frac{\pi}{a\left|\vec{v}-\vec{c}_{0}\right|} \int_{-\left|\vec{N}-\vec{c}_{0}\right|}^{\left|\vec{v}-\vec{c}_{0}\right|} e^{-a x^{2}} d x \\
& =\left(\frac{\pi}{a}\right)^{3 / 2} \frac{1}{\left|\vec{v}-\vec{c}_{0}\right|} \phi\left[\sqrt{a}\left|\vec{N}-\vec{c}_{0}\right|\right]
\end{aligned}
$$

2)

$$
\begin{aligned}
& I_{2}=\int_{-\infty}^{\infty} d^{3} \vec{v}_{1}\left|\vec{v}-\vec{v}_{1}\right| e^{-a\left(\vec{v}_{1}-\vec{c}_{0}\right)^{2}} \\
& =\int_{-\infty}^{\infty} d^{3} \vec{V}(V) e^{-a\left[\left(\vec{v}-\vec{c}_{0}\right)^{2}+V^{2}+2\left(\vec{v}-\vec{c}_{0}\right) \cdot \vec{v}\right]} \\
& =e^{-a\left(\vec{v}-\vec{c}_{0}\right)^{2}} \int_{0}^{\infty} v^{3} d V \int_{0}^{\pi} \sin \theta d \theta \int_{0}^{2 \pi} d \varphi-a\left[v^{2}+2\left(\vec{v}-\vec{c}_{0}\right) \cdot \vec{v}\right] \\
& =\frac{\pi}{a\left|\vec{v}-\vec{c}_{0}\right|} \int_{0}^{\infty} V^{2} e^{-a\left[\left|\vec{v}-\vec{c}_{0}\right|-V\right]^{2}} d V-\frac{\pi}{a\left|\vec{v}-\vec{c}_{0}\right|} \int_{0}^{\infty} V^{2} e^{-a\left[\left|\vec{v}-\vec{c}_{0}\right|+V\right]^{2}} d V \\
& =\frac{\pi}{a\left|\vec{v}-\vec{c}_{0}\right|} \int_{-\left|\vec{v}-\vec{c}_{0}\right|}^{\left|\vec{v}-\vec{c}_{0}\right|}\left[x^{2}+\left|\vec{v}-\vec{c}_{0}\right|^{2}\right] e^{-a x^{2}} d x \\
& +\frac{2 \pi}{a} \int_{-\left|\vec{N}-\vec{c}_{0}\right|}^{\infty} x e^{-a y^{2}} d y-\frac{2 \pi}{a} \int_{\infty}^{\left|\vec{v}-\vec{c}_{0}\right|} x e^{-a x^{2}} d x \\
& =\left(\frac{\pi}{a}\right)^{3 / 2}\left|\vec{N}-\vec{c}_{0}\right| \phi\left[\sqrt{a}\left|\vec{N}-\vec{c}_{0}\right|\right]+\frac{2 \pi}{a^{2}} e^{-a\left|\vec{N}-\vec{r}_{0}\right|^{2}} \\
& -\frac{\pi}{a^{2}} e^{-a\left|\vec{N}-\vec{r}_{0}\right|^{2}}+\frac{1}{2 a}\left(\frac{\pi}{a}\right)^{3 / 2} \frac{1}{\left|\vec{N}-\vec{c}_{0}\right|} \phi\left[\sqrt{a}\left|\vec{v}-\vec{c}_{0}\right|\right]
\end{aligned}
$$

92 


$$
=\frac{\pi}{a^{2}} e^{-a\left|\vec{v}-\vec{c}_{0}\right|^{2}}+\frac{1}{2 a}\left(\frac{\pi}{a}\right)^{3 / 2} \frac{1}{\left|\vec{v}-\vec{c}_{0}\right|} \phi\left[\sqrt{a}\left|\vec{v}-\vec{c}_{0}\right|\right]\left[1+2 a\left|\vec{v}-\vec{c}_{0}\right|^{2}\right]
$$

After substitution and simplification, this gives:

$$
\begin{aligned}
\left(\frac{\delta f_{3}}{\delta t}\right)_{C_{0} 11}=m \Gamma_{3}\left[-\frac{\partial}{\partial \vec{v}} \cdot\left\{f_{3}\right.\right. & \left.\frac{\partial}{\partial \vec{v}}\left[\left(\frac{M_{1}+M_{3}}{M_{1}}\right) \frac{1}{w_{1}} \phi\left(w_{1} \sqrt{a}\right)+\left(\frac{M_{2}+M_{3}}{M_{2}}\right) \frac{1}{w_{2}} \phi\left(w_{2} \sqrt{b}\right)\right]\right\} \\
+ & \frac{1}{2} \frac{\partial^{2}}{\partial \vec{v} \partial \vec{v}}:\left\{f _ { 3 } \frac { \partial ^ { 2 } } { \partial \vec { v } \cdot \vec { v } } \left[\frac{1}{4 a w_{1}}\left(4 w_{1} \sqrt{\frac{a}{n}} e^{-a w_{1}^{2}}+2\left(1+2 a w_{1}^{2}\right) \phi\left(w_{1} \sqrt{a}\right)\right)\right.\right. \\
& \left.\left.\left.+\frac{1}{4 b w^{2}}\left(4 w_{2} \sqrt{\frac{b}{n}} e^{-b w_{2}^{2}}+2\left(1+2 b w_{2}^{2}\right) \phi\left(w_{2} \sqrt{l}\right)\right)\right]\right\}\right]
\end{aligned}
$$


Allen, C. W., Repts. Progr. in Physics 17, 135 (1954)

Chamberlain, J., Astroph. J. 133, 675 (1961)

Chapman, S., Smithsonian Contrib. Astrophys. 2, 1 (1957)

Epstein, I., Ap. J. 114, 438 (1951)

Evans, J. W., The Solar Corona, Academic Press, New York and London, 1965

Flammarion, G. C., The Flammarion Book of Astronomy, English edition translated from the French edition of 1955-1960, brought up to date and first published 1964. Simon and Schuster, New York, 1964

Harrison, M. Hall, Ap. J. $\underline{108}, 310,1948$

Holt, E. H. and Haskell, R. E., Foundations of Plasma Dynamics, The Macmillan Company, New York, 1965

Jokipii, I. R., Effects of Diffusion on the Composition of the Solar Corona and the Solar Wind, Chapter XIV of "The Solar Wind," Pergamon Press, New York, 1966

Keller, M., Ap. J. 108, 310, 1948

Kuiper, G. P., The Sun, The University of Chicago Press, Chicago, 1953

Mackin, R. I., Jr. and Neugebauer, M., The Solar Wind, Pergamon Press, New York, 1966

Montgomery, D.C. and Tidman, D. A., Plasma Kinetic Theory, McGraw-Hill, New York, 1964

Nakada, M. P., Enhanced Evaporation and the Solar Wind, Unpublished Note 
Newkirk, G., I. A. U. Symposium No. 9: Radio Astron., p. 149 (1959)

Oke, J. B., Astrophys. J. 133, 166 (1961)

Pannekoek, A., Bull. Ast. Inst. Netherl. 1, 110 (1922)

Parker, E. N., Comments on Coronal Heating, Chapter in "The Solar Corona", Academic Press, New York and London, 1963

Parker, E. N., Interplanetary Dynamical Processes, Interscience Publishers, New York, 1963

Pottasch, S. R., Astrophys. J. 131, 68 (1960)

Rosseland, S., M. N. Roy. Astron. Soc. 84, 720-728 (1924)

Scarf, F. L. and Noble, L. M., Dynamics of the Solar Wind, Aerospace Science Meeting, New York, N. Y., Jan. 20-22, 1964

Schwarzschild, M., Structure and Evolution of Stars, Princeton University Press, Princeton, 1958

Schwarzschild, M., Ap. J. 104, 203 (1946)

Spitzer, L. Jr., Physics of Fully Ionized Gases, 2nd edition, Interscience Publishers, New York, 1962

Sturrock, P. A. and Hartle, R. E., Phys. Rev. Letters, $\underline{16}, 628-631$ (1966)

Unsöld, A., Z. Astrophys. $\underline{45}, 113$ (1958)

Van de Hulst, H. C., The Chromosphere and the Corona, chapter in "The Sun", edited by G. P. Kuiper, The University of Chicago Press, Chicago, 1953

Whang, Y. C., Liu, C. K., and Chang, C. C., Astroph. J. $\underline{145}, 255$ (1966)

Wrubel, M. H, Stellar Interiors. Handbuck der Physik, vol. 51, p. 1, Springer, Berlin, 1958 